\title{
ACOUSTIC SCATTERING BY MILDLY ROUGH UNBOUNDED SURFACES IN THREE DIMENSIONS*
}

\author{
SIMON N. CHANDLER-WILDE ${ }^{\dagger}$, ERIC HEINEMEYER ${ }^{\ddagger}$, AND ROLAND POTTHAST ${ }^{\ddagger}$
}

\begin{abstract}
For a nonlocally perturbed half-space we consider the scattering of time-harmonic acoustic waves. A second kind boundary integral equation formulation is proposed for the sound-soft case, based on a standard ansatz as a combined single- and double-layer potential but replacing the usual fundamental solution of the Helmholtz equation with an appropriate half-space Green's function. Due to the unboundedness of the surface, the integral operators are noncompact. In contrast to the two-dimensional case, the integral operators are also strongly singular, due to the slow decay at infinity of the fundamental solution of the three-dimensional Helmholtz equation. In the case when the surface is sufficiently smooth (Lyapunov) we show that the integral operators are nevertheless bounded as operators on $L^{2}(\Gamma)$ and on $L^{2}(\Gamma) \cap B C(\Gamma)$ and that the operators depend continuously in norm on the wave number and on $\Gamma$. We further show that for mild roughness, i.e., a surface $\Gamma$ which does not differ too much from a plane, the boundary integral equation is uniquely solvable in the space $L^{2}(\Gamma) \cap B C(\Gamma)$ and the scattering problem has a unique solution which satisfies a limiting absorption principle in the case of real wave number.
\end{abstract}

Key words. boundary integral equation method, rough surface scattering, Helmholtz equation

AMS subject classifications. 35J05, 35J25, 45E10, 45E99, 78A45

DOI. $10.1137 / 050635262$

1. Introduction. The simulation of scattering of acoustic or electromagnetic waves is of great importance for a large number of application areas ranging from medical imaging to seismic exploration. To carry out this simulation, boundary integral equation (BIE) methods have become very popular in recent decades. For scattering by bounded obstacles in two or three dimensions a very complete theory of the boundary integral equation method has been developed (e.g., [14, 20]), and the method forms the basis of very effective numerical algorithms (e.g., [12]).

This paper is concerned with the problem of scattering by unbounded surfaces for which the mathematical theory is much less well developed. More precisely, we are concerned with what are termed rough surface scattering problems in the engineering literature. We use the phrase rough surface, as is the practice in this literature, to denote a surface which is a (usually nonlocal) perturbation of an infinite plane surface such that the whole surface lies within a finite distance of the original plane. In particular we have in mind what is the usual case in the engineering literature where the scattering surface $\Gamma$ is the graph of some bounded continuous function $f: \mathbb{R}^{2} \rightarrow \mathbb{R}$, i.e.,

$$
\Gamma:=\left\{x=\left(x_{1}, x_{2}, x_{3}\right) \in \mathbb{R}^{3}: x_{3}=f\left(x_{1}, x_{2}\right)\right\} .
$$

We will focus on a typical problem of this type, namely acoustic scattering by a rough, sound soft surface, the acoustic medium of propagation occupying the perturbed half-

\footnotetext{
* Received by the editors July 6, 2005; accepted for publication (in revised form) November 29, 2005; published electronically March 3, 2006.

http://www.siam.org/journals/siap/66-3/63526.html

${ }^{\dagger}$ Department of Mathematics, University of Reading, Whiteknights, P.O. Box 220, Berkshire RG6 6AX, UK (s.n.chandler-wilde@reading.ac.uk).

$\ddagger$ Institute for Numerical and Applied Mathematics, University of Göttingen, Lotzestr. 16-18, 37083 Göttingen, Germany (potthast@scienceatlas.de, heinemey@math.uni-goettingen.de).
} 
space

$$
D:=\left\{x=\left(x_{1}, x_{2}, x_{3}\right): x_{3}>f\left(x_{1}, x_{2}\right)\right\}
$$

above the scattering surface $\Gamma$. The paper is concerned, particularly, with the theory of BIE methods for such problems, in the case when $f$ is a sufficiently smooth function ( $\Gamma$ is Lyapunov).

Rough surface scattering problems arise frequently in applications, for example modelling acoustic and electromagnetic wave propagation over outdoor ground and sea surfaces or, at a very different scale, optical scattering from the surface of materials in nanotechnology. The mathematical and computational modelling of these problems has a large literature; see, e.g., the reviews and monographs by Ogilvy [22], Voronovich [28], Saillard and Sentenac [25], Warnick and Chew [29], and DeSanto [15]. The simulation of these scattering problems, requiring discretizations of sections of three-dimensional (3D) surfaces of diameter large compared to the wavelength, is a substantial scientific computing problem for which BIE methods are very popular, with many effective, specialized numerical algorithms developed [27, 25, 29, 31].

Although BIE methods are applied widely to rough surface scattering problems, the mathematical basis of the method is still poorly developed, especially in the 3D case. In fact, there are a number of severe difficulties in extending the theory of BIE methods from bounded to unbounded scatterers.

The first of these difficulties is that, due to the slow decay at infinity of the standard fundamental solution, $\Phi(x, y)$, of the Helmholtz equation (like $|x-y|^{-(n-1) / 2}$ in $n$ dimensions), the standard boundary integral operators are not bounded on any of the standard function spaces when the surface is unbounded. We will see that this difficulty can be overcome by modifying the usual kernels so as to obtain bounded integral operators and corresponding novel BIE formulations.

A second difficulty is that of loss of compactness of boundary integral operators associated with the noncompactness of the unbounded scattering surface. This is a severe barrier to establishing existence of solution to the BIEs. We recall that, in the case of scattering by smooth bounded obstacles, compactness arguments (the RieszFredholm theory) lead directly to proofs of well-posedness for second kind boundary integral equation formulations (e.g., [14]). In the case of nonsmooth (Lipschitz) obstacles, compactness arguments are no longer sufficient but still play an essential role in establishing well-posedness (e.g., [26]).

For the two-dimensional (2D) rough surface scattering case much progress has been made in terms of deriving well-posed BIEs for a variety of acoustic, electromagnetic, and elastic wave problems $[9,8,32,2]$. Surprisingly, none of the analysis for the $2 \mathrm{D}$ case extends straightforwardly to three dimensions; indeed most of the $2 \mathrm{D}$ analysis appears to be unsuitable in the 3D case.

In more detail, in the $2 \mathrm{D}$ case bounded integral operators have been obtained by replacing the standard fundamental solution by the Dirichlet or impedance Green's function for a half-plane that contains the domain $D$ of propagation (see, e.g., $[8,32]$ ). This modification leads to kernels of boundary integral operators that are weakly singular in their asymptotic behavior at infinity so that the integral operators are bounded on $L^{p}(\Gamma)$ for $1 \leq p \leq \infty$ and on $B C(\Gamma)$, the space of bounded continuous functions on $\Gamma$. In this paper we will employ the analogous modification for the $3 \mathrm{D}$ case, replacing the standard fundamental solution with the Dirichlet Green's function for a half-space that contains $D$. But this modification leads to kernels of the integral operators that are strongly rather than weakly singular. As a consequence, 


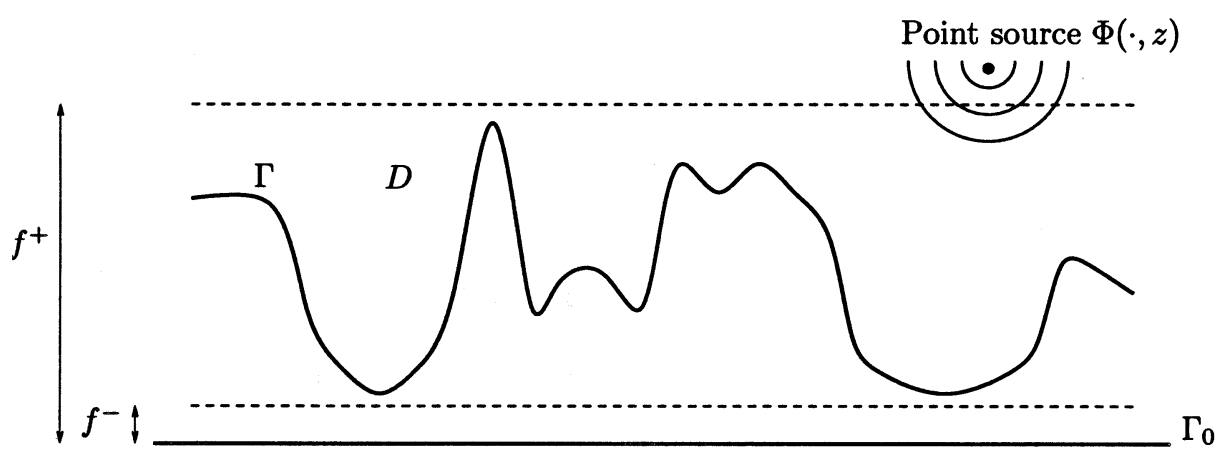

FIG. 1.1. Geometrical setting of the scattering problem.

the boundary integral operators are no longer well defined as operators on $B C(\Gamma)$ or $L^{\infty}(\Gamma)$. We are, however, able to show the boundedness of the operators on $L^{2}(\Gamma)$ by more elaborate arguments, expressing each integral operator as the sum of products of convolution and multiplication operators plus a well-behaved remainder, each multiplication operator a multiplication by an $L^{\infty}$ function. To complete the proof of boundedness of the integral operators, one of the main results of the paper, we show, by explicit computations, that the Fourier transform of each convolution kernel is bounded. We note that our technique of expressing the kernel as the sum of products of convolution and multiplication operators plus a short-range remainder has been used previously $[27,31]$ but as a computational rather than a theoretical tool, as a device for matrix compression and acceleration of matrix-vector multiplications in iterative solvers.

To establish existence of solution and well-posedness in the 2D case, generalizations of part of the Riesz theory of compact operators have been developed [24, 11, 10] which require only local compactness rather than compactness and enable existence of solution in $B C(\Gamma)$ to be deduced from uniqueness of solution. In fact, injectivity of the second kind BIE in $B C(\Gamma)$ implies well-posedness in $B C(\Gamma)$ and in the space $L^{p}(\Gamma), 1 \leq p \leq \infty[3]$. But this theory does not seem relevant for 3D rough surface scattering problems given that the corresponding boundary integral operators are not well defined as operators on $B C(\Gamma)$. In the absence of these tools we will prove existence of solution to the BIE (and the corresponding scattering problem) by perturbation arguments, used for the much simpler $2 \mathrm{D}$ case in [7]. The perturbation arguments we employ will prove to be sufficient to establish existence in the case when $\Gamma$ is sufficiently close to a flat plane.

The results contained in this paper are as follows. We suppose that the rough surface is given by (1.1) with $f$ continuously differentiable with Hölder continuous first derivative $\left(f \in B C^{1, \alpha}\left(\mathbb{R}^{2}\right)\right.$ for some $\left.\alpha \in(0,1]\right)$. As mentioned above, we investigate the mapping properties of the single- and double-layer potentials when the kernel is the Dirichlet Green's function for a half-space, consisting of the standard fundamental solution minus the same function with a point source mirrored in a plane. By Fourier techniques on a 2D plane and appropriate decompositions of the operators into a local and a global part we show that these layer potentials exist as bounded operators on $L^{2}(\Gamma)$. After these results, of significant interest in their own right, we consider the problem of acoustic scattering by the rough surface $\Gamma$ in the case when the surface is sound soft (the field vanishes on $\Gamma$ ) and the incident field is due to a source distribution 
with compact support in $D$. We reduce this scattering problem to a second kind boundary integral equation via an ansatz for the solution as a combined single- and double-layer potential. (The analogous ansatz was used for the $2 \mathrm{D}$ rough surface scattering case in [32], based on the analogous approach for scattering by bounded obstacles dating back to [4].) For a flat surface, unique solvability of the BIE is shown by explicit computation of a symbol. We then prove continuous dependence of the boundary integral operators on variations of the boundary and use these results and perturbation arguments to show that the scattering problem has a solution for all surfaces in a neighborhood of the plane, i.e., for mildly rough unbounded surfaces. Moreover, we show that the solution we compute by the BIE method satisfies a limiting absorption principle. For the convenience of the reader our main results are collected together and precisely stated at the end of section 2 .

We should point out that a rigorous mathematical theory for BIE methods for $3 \mathrm{D}$ rough surface scattering has been developed previously for two special cases, both instances where the integral equation can be reduced to one on a finite domain so that compactness arguments can be applied. The first is the case of scattering by a locally perturbed plane, where the unbounded surface coincides with a plane in the exterior of some ball. This case can be reduced to a BIE on a finite domain, related to the local perturbation; we refer the reader to $[30,19,5]$ and the references therein. The second is the case when the surface is a diffraction grating (the function $f$ in (1.1) is biperiodic) and the incident field is a plane wave. In this case the BIE can be reduced to one on a finite part of the surface that is a single period; see [21, 17].

Finally, we note that, since our results assume boundary data in the space $L^{2}(\Gamma)$, they do not include the interesting and problematic case of plane wave incidence, which is included in the analogous theory that has been developed for the $2 \mathrm{D}$ problem $[8,32]$. For a partial theoretical justification for BIE methods for 3D rough surface scattering with plane wave incidence, namely a justification, with some provisos, of Green's representation formula, see [16].

Notation. Throughout the paper $x$ and $y$ will denote points in $\mathbb{R}^{3}$ with components $x=\left(x_{1}, x_{2}, x_{3}\right)$ and $y=\left(y_{1}, y_{2}, y_{3}\right)$. The reflection of $y \in \mathbb{R}^{3}$ in the plane $\Gamma_{0}:=\left\{x \in \mathbb{R}^{3}: x_{3}=0\right\}$ will be denoted by $y^{\prime}:=\left(y_{1}, y_{2},-y_{3}\right)$. By $\mathbf{x}$ we will denote $\left(x_{1}, x_{2}\right) \in \mathbb{R}^{2}$, as well as the projection $\left(x_{1}, x_{2}, 0\right)$ of $x$ onto the plane $\Gamma_{0}$. Similarly $\mathbf{y}$ denotes $\left(y_{1}, y_{2}\right)$ and the projection of $y$ onto $\Gamma_{0}$. The standard scalar product in $\mathbb{R}^{2}$ is denoted by $\mathbf{x} \cdot \mathbf{y}$ and $|\cdot|$ is the Euclidean norm in $\mathbb{R}^{n}$. Let $H^{+}:=\{z \in \mathbb{C}: \operatorname{Im} z \geq 0, \operatorname{Re} z>0\}$. Given an unbounded closed set $S \subset \mathbb{R}^{n}, n=2,3$, $B C(S)$ will denote the set of bounded continuous real- or complex-valued functions on $S$, a Banach space with the norm $\|\cdot\|_{B C(S)}$ defined by $\|F\|_{B C(S)}=\sup _{x \in S}|F(x)|$. We will employ this notation particularly often in the cases $S=\Gamma \subset \mathbb{R}^{3}$ and $S=\mathbb{R}^{2}$. Similarly, for $0<\alpha \leq 1$, let $B C^{1, \alpha}\left(\mathbb{R}^{2}\right)$ denote the set of those bounded continuously differentiable functions $F: \mathbb{R}^{2} \rightarrow \mathbb{R}$ that have the property that $\nabla F$ is bounded and uniformly Hölder continuous with index $\alpha$, so that

$$
\left.|| F\right|_{B C^{1, \alpha}\left(\mathbb{R}^{2}\right)}:=\sup _{\mathbf{x} \in \mathbb{R}^{2}}|F(\mathbf{x})|+\sup _{\mathbf{x} \in \mathbb{R}^{2}}|\nabla F(\mathbf{x})|+\sup _{\mathbf{x}, \mathbf{y} \in \mathbb{R}^{2}, \mathbf{x} \neq \mathbf{y}} \frac{|\nabla F(\mathbf{x})-\nabla F(\mathbf{y})|}{|\mathbf{x}-\mathbf{y}|^{\alpha}}<\infty .
$$

$B C^{1, \alpha}\left(\mathbb{R}^{2}\right)$ is a Banach space under the norm $\|\cdot\|_{B C^{1, \alpha}\left(\mathbb{R}^{2}\right)}$. It is convenient also to have a shorthand for the intersection of the sets $L^{2}(\Gamma)$ and $B C(\Gamma)$, so we define

$$
X:=L^{2}(\Gamma) \cap B C(\Gamma)
$$


Since $L^{2}(\Gamma)$ and $B C(\Gamma)$ are Banach spaces equipped with their respective norms, so also is $X$, equipped with the norm $\|\cdot\|_{X}$ defined by

$$
\|F\|_{X}:=\max \left(\|F\|_{L^{2}(\Gamma)},\|F\|_{B C(\Gamma)}\right) .
$$

2. Scattering by rough surfaces in $\mathbb{R}^{3}$. Time-harmonic ( $e^{-i \omega t}$ time dependence) acoustic waves are modelled by the Helmholtz equation

$$
\triangle u+\kappa^{2} u=0
$$

where $\kappa=\kappa_{0}+i \kappa_{1}$ denotes the wave number for which we will assume that $\kappa \in H^{+}$, i.e., $\kappa_{0}>0$ and $\kappa_{1} \geq 0$. We define the domain of propagation $D$ by (1.2), where $f \in$ $B C^{1, \alpha}\left(\mathbb{R}^{2}\right)$ is a strictly positive function, so that there exist constants $f^{+}>f^{-}>0$ with

$$
f^{-} \leq f(\mathbf{x}) \leq f^{+}, \quad \mathbf{x} \in \mathbb{R}^{2}
$$

We denote the boundary of $D$ by $\Gamma$, so that $\Gamma$ is given by (1.1). (For a sketch of the geometry see Figure 1.1.) Whenever we wish to denote explicitly the dependence of the domain on the boundary function $f$ we will write $D_{f}$ for $D$ and $\Gamma_{f}$ for $\Gamma$. This of course includes the case of the constant function $f \equiv h \in \mathbb{R}^{+}:=(0, \infty)$.

We will consider the scattering of an incident acoustic wave $u^{i}$ by the surface $\Gamma$. For the total field

$$
u:=u^{i}+u^{s}
$$

which is the sum of the incident field and the scattered field $u^{s}$, we assume on $\Gamma$ the Dirichlet boundary condition

$$
u(x)=0, \quad x \in \Gamma .
$$

We require that the scattered field is bounded in $D$, i.e.,

$$
\left|u^{s}(x)\right| \leq c, \quad x \in D,
$$

for some constant $c>0$. In the case $\kappa>0$ we also require that $u$ satisfies the following limiting absorption principle: denoting $u$ temporarily by $u^{(\kappa)}$ to indicate its dependence on $\kappa$, we suppose that for all sufficiently small $\epsilon>0$ a solution $u^{(\kappa+i \epsilon)}$ exists and that, for all $x \in D$,

$$
u^{(\kappa+i \epsilon)}(x) \rightarrow u^{(\kappa)}(x), \quad \epsilon \rightarrow 0 .
$$

The limiting absorption principle plays the role of a radiation condition for real $\kappa$ to single out the physical solution.

Before proceeding further to define the scattering problem precisely, we want to take a look, in the important case when the wave number is real, at the fundamental solution

$$
\Phi(x, y):=\frac{1}{4 \pi} \frac{e^{i \kappa|x-y|}}{|x-y|}, \quad x, y \in \mathbb{R}^{3}, x \neq y,
$$

of the Helmholtz equation in $\mathbb{R}^{3}$ and the ordinary boundary layer potentials, e.g., the single-layer potential

$$
\int_{\Gamma} \Phi(x, y) \varphi(y) d s(y), \quad x \in \mathbb{R}^{3} .
$$


For an unbounded surface $\Gamma$ the integral (2.7) converges (for $\kappa>0$ ) only if $\varphi$ decreases sufficiently rapidly at infinity. This is due to the slow decay of the fundamental solution in $\mathbb{R}^{3}$ at infinity. Letting $B_{R}(x):=\left\{y \in \mathbb{R}^{3}:|x-y|<R\right\}$ denote the open ball of radius $R$ centered at $x$, an easy calculation yields that, for $x \in D, p>0$, and $\kappa>0$,

$$
\int_{\Gamma \cap B_{R}(x)}|\Phi(x, y)|^{p} d s(y)=\int_{\Gamma \cap B_{R}(x)} \frac{1}{|x-y|^{p}} d s(y) \rightarrow \infty, \quad R \rightarrow \infty,
$$

for $p \leq 2$. Thus we observe that the trace of $\Phi(x, \cdot)$ on $\Gamma$ is not integrable; indeed $\Phi(x, \cdot) \notin L^{p}(\Gamma)$ for $p \leq 2$. Thus, for every $x \in D$, the single-layer potential (2.7) is not well defined for all $\varphi \in L^{2}(\Gamma)$.

In order to get a faster decaying kernel we will, following what has been proposed for the analogous $2 \mathrm{D}$ rough surface scattering case [32], replace $\Phi(x, y)$ by an appropriate half-space Green's function for the Helmholtz equation. Specifically, we will work with the function

$$
G(x, y):=\Phi(x, y)-\Phi\left(x, y^{\prime}\right),
$$

with $y^{\prime}=\left(y_{1}, y_{2},-y_{3}\right)$, which is the Dirichlet Green's function for the half-space $\left\{x: x_{3}>0\right\}$. Thus we will use layer potentials with $\Phi(x, y)$ replaced by $G(x, y)$, so that we define the single-layer potential operator by

$$
(S \varphi)(x):=2 \int_{\Gamma} G(x, y) \varphi(y) d s(y), \quad x \in \Gamma,
$$

and the double-layer potential operator by

$$
(K \varphi)(x):=2 \int_{\Gamma} \frac{\partial G(x, y)}{\partial \nu(y)} \varphi(y) d s(y), \quad x \in \Gamma,
$$

where the normal $\nu(y)$ is directed into $D$. Whenever we wish to denote explicitly the dependence of $S$ and $K$ on the boundary function $f$ we will write $S_{f}$ and $K_{f}$ for $S$ and $K$, respectively.

It is a straightforward calculation (cf. (3.7) below) to see that, for $y \in \Gamma$,

$$
|G(x, y)| \sim \frac{x_{3} y_{3}|\kappa|}{2 \pi} \frac{e^{-\kappa_{1}|x-y|}}{|x-y|^{2}}, \quad|y| \rightarrow \infty .
$$

This decay and the analogous decay at infinity (3.11) that we show for the kernel of the double-layer potential operator are fast enough for (2.9) and (2.10) to be well defined as improper integrals, for every $x \in \bar{D}$ and $\varphi \in C(\Gamma) \cap L^{2}(\Gamma)$, in particular in the case $\kappa_{1}=0$. Further, we will show, via Fourier techniques in section 5 , as a main result of the paper, that this decay is fast enough for $S$ and $K$ to be bounded operators on $L^{2}(\Gamma)$.

Because, for $x \in \Gamma$,

$$
\int_{\Gamma \cap B_{R}(x) \backslash B_{1}(x)} \frac{1}{|x-y|^{2}} d s(y) \rightarrow \infty, \quad R \rightarrow \infty,
$$

the decay of $G(x, y)$ as $y \rightarrow \infty$ is not fast enough when $\kappa>0$ for $S$ to be well defined as an operator on the space of bounded continuous functions. Thus integral equation 
methods for the 3D rough surface scattering problem are essentially different from the $2 \mathrm{D}$ case studied in $[7,9,8,32,3]$.

Returning to the scattering problem, we wish to develop an analysis that is applicable whenever the incident wave is due to sources of the acoustic field located in some compact set $M \subset D$. Since waves with sources in a bounded set $M \subset \mathbb{R}^{3}$ can be represented as superpositions of point sources located in the same set, we will concentrate on the case when the incident field is due to a point source located at some point $z \in D$, i.e., $u^{i}=\Phi(\cdot, z)$. Thus the following is the specific problem that we will consider in this paper:

Problem 1 (point source rough surface scattering problem). Let $u^{i}=\Phi(\cdot, z)$ be the incident field due to a point source at $z \in D$. Then we seek a scattered field $u^{s} \in C^{2}(D) \cap C(\bar{D})$ such that $u^{s}$ is a solution to the Helmholtz equation (2.1) in D, the total field satisfies the sound-soft boundary condition (2.3), and the bound (2.4) holds. In the case $\kappa>0$, we also require that the limiting absorption principle (2.5) holds.

We will convert this scattering problem to a boundary value problem. To do this we will seek the scattered field as the sum of a mirrored point source $\Phi^{\prime}(\cdot, z):=$ $-\Phi\left(\cdot, z^{\prime}\right)$, where $z^{\prime}$ is the reflection of $z$ in the flat plane $\Gamma_{0}$, plus some unknown remainder $v$, i.e., $u^{s}=v+\Phi^{\prime}(\cdot, z)$. Note that $\Phi^{\prime}(\cdot, z)$ is a solution to the scattering problem in the special case that $\Gamma=\Gamma_{0}$. Using the boundary condition $u^{s}+\Phi(\cdot, z)=0$ on $\Gamma=\partial D$, we obtain the boundary condition on $v$ that

$$
v(x)=-\left\{\Phi(x, z)-\Phi\left(x, z^{\prime}\right)\right\}=-G(x, z)=: g(x), \quad x \in \Gamma .
$$

Clearly, $g \in B C(\Gamma)$ and it follows from (2.11) that $g \in L^{2}(\Gamma)$, so that $g \in X=$ $L^{2}(\Gamma) \cap B C(\Gamma)$. Thus $u^{s}$ satisfies the above scattering problem if and only if $v$ satisfies the following Dirichlet problem with $g$ given by (2.12).

Problem 2 (BVP). Given $g \in X$, find $v \in C^{2}(D) \cap C(\bar{D})$, which satisfies the Helmholtz equation (2.1) in D, the Dirichlet boundary condition $v=g$ on $\Gamma$, the bound (2.4), and, for $\kappa>0$, the limiting absorption principle (2.5).

In this paper we will look for a solution to this boundary value problem as the combined single- and double-layer potential

$$
v(x):=u_{2}(x)-i \eta u_{1}(x), \quad x \in D,
$$

with some parameter $\eta \geq 0$, where for a given function $\varphi \in X$ we define the singlelayer potential

$$
u_{1}(x):=\int_{\Gamma} G(x, y) \varphi(y) d s(y), \quad x \in \mathbb{R}^{3},
$$

and the double-layer potential

$$
u_{2}(x):=\int_{\Gamma} \frac{\partial G(x, y)}{\partial \nu(y)} \varphi(y) d s(y), \quad x \in \mathbb{R}^{3} .
$$

Seeking the solution in this form we will see that the boundary condition (2.12) is satisfied if and only if the BIE

$$
(I+K-i \eta S) \varphi=2 g
$$

holds on $\Gamma$, where $I$ is the identity operator. 
The following are the main results in the remainder of the paper. In the next two sections we examine the kernels of the integral operators $K$ and $S$ and recall relevant properties of convolution and more general integral operators that we need to study $K$ and $S$. The properties we discuss are exploited in section 5 . In particular we show the following result.

THEOREM 2.1. The single- and double-layer potential operators $S$ and $K$, defined by (2.9) and (2.10), are bounded operators on $L^{2}(\Gamma)$ and on $X$.

We also establish that the single- and double-layer potential operators $S$ and $K$ depend continuously on $\kappa_{1}=\operatorname{Im} \kappa$ in the norm topology on the set of bounded linear operators on $L^{2}(\Gamma)$; this result is needed to establish the limiting absorption principle. Moreover, we show, for $S$ and $K$, continuous dependence in norm on the boundary $\Gamma$, in a sense we make precise.

In the final section, section 6 , we establish existence and uniqueness of solution of the BIE and boundary value problem, at least in certain cases. As the first step we justify the integral equation (2.16) as a reformulation of the boundary value problem, showing the following result.

Theorem 2.2. Suppose that $v$ is defined by (2.13)-(2.15) with $\varphi \in X$. Then, in the case $\kappa_{1}>0, v$ satisfies the boundary value problem if and only if $\varphi$ satisfies the BIE (2.16). In the case $\kappa_{1}=0$ (i.e., $\kappa>0$ ), if $v$ satisfies the boundary value problem, then $\varphi$ satisfies (2.16). Conversely, if $\kappa>0, \varphi^{(\kappa+i \epsilon)} \in X$ satisfies the integral equation (2.16) with $\kappa$ replaced by $\kappa+i \epsilon$, for all sufficiently small $\epsilon>0$, and $\left\|\varphi-\varphi^{(\kappa+i \epsilon)}\right\|_{L_{2}(\Gamma)} \rightarrow 0$ as $\epsilon \rightarrow 0$, then $v$ satisfies the boundary value problem.

We further establish the following result.

THEOREM 2.3. The boundary value problem has at most one solution.

Then we study the invertibility of the operator $I+K-i \eta S$, first for the case when $\Gamma$ is flat and the operator $I+K-i \eta S$ is a convolution operator and then for the case when $\Gamma$ is mildly rough by perturbation arguments. Our main result is the following.

THEOREM 2.4. Suppose that $h>0$ and that either $\eta>0$ or $\eta=0$ and $\operatorname{Im} \kappa=$ $\kappa_{1}>0$. Then, provided $\|f-h\|_{B C^{1, \alpha}\left(\mathbb{R}^{2}\right)}$ is sufficiently small (so that $\Gamma_{f}$ is sufficiently close to the flat surface $f \equiv h$ ), it holds that the integral equation (2.16) has a unique solution $\varphi \in L^{2}(\Gamma)$ for every $g \in L^{2}(\Gamma)$, so that $(I+K-i \eta S)^{-1}$ exists and is bounded as an operator on $L^{2}(\Gamma)$. If, further, $g \in X$, then $\varphi \in X$, so that $(I+K-i \eta S)^{-1}$ is also a bounded operator on $X$.

Combining these results we have a final corollary concerning the solvability of the boundary value problem.

THEOREM 2.5. If $h>0$ and $\|f-h\|_{B C^{1, \alpha}\left(\mathbb{R}^{2}\right)}$ is sufficiently small (so that $\Gamma_{f}$ is sufficiently close to the flat surface $f \equiv h$ ), then the boundary value problem has exactly one solution. Further, for some constant $c>0$, independent of $g$,

$$
|v(x)| \leq c\|g\|_{X}, \quad x \in \bar{D} .
$$

3. Properties of the 3D fundamental solution. We start with an investigation of properties of the fundamental solution $\Phi(x, y)$ and its derivatives. The key results are the expansions (3.7) and (3.11) needed to prove mapping properties of the boundary integral operators $S$ and $K$ in section 5 .

For the first derivative of $\Phi(x, y)$ with respect to $y_{3}$ we calculate

$$
\frac{\partial \Phi(x, y)}{\partial y_{3}}=-\frac{i \kappa}{4 \pi} \frac{\left(x_{3}-y_{3}\right)}{|x-y|^{2}} e^{i \kappa|x-y|}+\frac{1}{4 \pi} \frac{\left(x_{3}-y_{3}\right)}{|x-y|^{3}} e^{i \kappa|x-y|} .
$$


The second derivative is given by

$$
\begin{aligned}
\frac{\partial^{2} \Phi(x, y)}{\partial y_{3}^{2}}=\frac{1}{4 \pi}\{ & i \kappa \frac{e^{i \kappa|x-y|}}{|x-y|^{2}}-\kappa^{2} \frac{\left(x_{3}-y_{3}\right)^{2}}{|x-y|^{3}} e^{i \kappa|x-y|}-2 i \kappa \frac{\left(x_{3}-y_{3}\right)^{2}}{|x-y|^{4}} e^{i \kappa|x-y|} \\
& \left.-\frac{e^{i \kappa|x-y|}}{|x-y|^{3}}-i \kappa \frac{\left(x_{3}-y_{3}\right)^{2}}{|x-y|^{4}} e^{i \kappa|x-y|}+3 \frac{\left(x_{3}-y_{3}\right)^{2}}{|x-y|^{5}} e^{i \kappa|x-y|}\right\} .
\end{aligned}
$$

For the third derivative with respect to $y_{3}$ we obtain

$$
\frac{\partial^{3} \Phi(x, y)}{\partial y_{3}^{3}}=\frac{3 \kappa^{2}}{4 \pi} \frac{\left(x_{3}-y_{3}\right)}{|x-y|^{3}} e^{i \kappa|x-y|}+O\left(\frac{1}{|x-y|^{4}}\right) .
$$

This holds in the sense that, given $c>0$ and a compact subset $S$ of $H^{+}$, there exists a constant $C>0$ such that

$$
\left|\frac{\partial^{3} \Phi(x, y)}{\partial y_{3}^{3}}-\frac{3 \kappa^{2}}{4 \pi} \frac{\left(x_{3}-y_{3}\right)}{|x-y|^{3}} e^{i \kappa|x-y|}\right| \leq \frac{C}{|x-y|^{4}}
$$

for all $x, y \in \mathbb{R}^{3}, x \neq y$, with $x_{3}, y_{3} \in[0, c]$ and all $\kappa \in S$. The similar equations below, in particular (3.7) and (3.11), are to be understood in an analogous fashion.

We use Taylor's expansion for the fundamental solution $\Phi(x, y)$ with respect to variations of $x_{3}$ and $y_{3}$. From Taylor's theorem, if $g \in C^{3}[0, \infty)$, then

$$
g(s)=g(0)+g^{\prime}(0) s+\frac{1}{2} g^{(2)}(0) s^{2}+\frac{1}{3 !} \int_{0}^{s}(s-t)^{2} g^{(3)}(t) d t, \quad s>0 .
$$

Applying (3.4) to $g(s):=\Phi\left(x, \mathbf{y}+s e_{3}\right)$, where $e_{3}$ is the unit vector in the $x_{3}$-direction, with $\mathbf{y}=\left(y_{1}, y_{2}, 0\right) \in \Gamma_{0}$ and $s \in[0, c]$ with some constant $c$, we obtain

$$
\begin{aligned}
\Phi\left(x, \mathbf{y}+s e_{3}\right)= & \frac{1}{4 \pi} \frac{e^{i \kappa|x-\mathbf{y}|}}{|x-\mathbf{y}|}-\frac{i \kappa}{4 \pi} \frac{x_{3} e^{i \kappa|x-\mathbf{y}|}}{|x-\mathbf{y}|^{2}} s \\
& +\frac{i \kappa}{4 \pi} \frac{e^{i \kappa|x-\mathbf{y}|}}{|x-\mathbf{y}|^{2}} \frac{s^{2}}{2}+O\left(\frac{1}{|x-\mathbf{y}|^{3}}\right) .
\end{aligned}
$$

To estimate the properties of single- and double-layer potentials on $L^{2}(\Gamma)$ we need to use Taylor's expansion also with respect to $x_{3}$. We treat all the terms of (3.5) separately and obtain, after some calculations,

$$
\begin{aligned}
\Phi\left(\mathbf{x}+h e_{3}, \mathbf{y}+s e_{3}\right)= & \frac{1}{4 \pi} \frac{e^{i \kappa|\mathbf{x}-\mathbf{y}|}}{|\mathbf{x}-\mathbf{y}|} \\
& +\frac{1}{4 \pi} \frac{i \kappa e^{i \kappa|\mathbf{x}-\mathbf{y}|}}{|\mathbf{x}-\mathbf{y}|^{2}} \frac{(h-s)^{2}}{2}+O\left(\frac{1}{|\mathbf{x}-\mathbf{y}|^{3}}\right) .
\end{aligned}
$$

Altogether we obtain

$$
G\left(\mathbf{x}+h e_{3}, \mathbf{y}+s e_{3}\right)=-\frac{1}{4 \pi} \frac{i \kappa e^{i \kappa|\mathbf{x}-\mathbf{y}|}}{|\mathbf{x}-\mathbf{y}|^{2}} 2 h s+O\left(\frac{1}{|\mathbf{x}-\mathbf{y}|^{3}}\right),
$$

in the sense that, given $c>0$ and a compact subset $S$ of $H^{+}$, there exists a constant $C>0$ such that

$$
\left|G\left(\mathbf{x}+h e_{3}, \mathbf{y}+s e_{3}\right)+\frac{2 h s}{4 \pi} \frac{i \kappa e^{i \kappa|\mathbf{x}-\mathbf{y}|}}{|\mathbf{x}-\mathbf{y}|^{2}}\right| \leq \frac{C}{|\mathbf{x}-\mathbf{y}|^{3}}
$$


for all $\mathbf{x}, \mathbf{y} \in \mathbb{R}^{2}$ with $\mathbf{x} \neq \mathbf{y}$, all $\kappa \in S$, and all $h, s \in[0, c]$. Arguing precisely as in [7] in the case $|x-y|>1$, we can also show the bound that (cf. [7, equations (3.6) and (3.8)]), given a compact subset $S \subset H^{+}$, there exists a constant $C>0$ such that

$$
|G(x, y)| \leq \frac{C\left(1+x_{3}\right)\left(1+y_{3}\right)}{|x-y|^{2}}
$$

for all $x, y \in \mathbb{R}^{3}$ with $x, y \neq 0$ and $x_{3}, y_{3} \geq 0$ and all $\kappa \in S$.

For the normal derivative of $G$, noting that $\partial \Phi\left(x, y^{\prime}\right) / \partial \nu(y)=\partial \Phi\left(x^{\prime}, y\right) / \partial \nu(y)$ and introducing the notation $\boldsymbol{\nu}(y):=\left(\nu_{1}(y), \nu_{2}(y)\right)$, we derive

$$
\begin{aligned}
4 \pi \frac{\partial G(x, y)}{\partial \nu(y)}= & -i \kappa \boldsymbol{\nu}(y) \cdot(\mathbf{x}-\mathbf{y})\left\{\frac{e^{i \kappa|x-y|}}{|x-y|^{2}}-\frac{e^{i \kappa\left|x-y^{\prime}\right|}}{\left|x-y^{\prime}\right|^{2}}\right\} \\
& +\boldsymbol{\nu}(y) \cdot(\mathbf{x}-\mathbf{y})\left\{\frac{e^{i \kappa|x-y|}}{|x-y|^{3}}-\frac{e^{i \kappa\left|x-y^{\prime}\right|}}{\left|x-y^{\prime}\right|^{3}}\right\} \\
& -i \kappa \frac{\nu_{3}(y)\left(x_{3}-y_{3}\right)}{|x-y|^{2}} e^{i \kappa|x-y|}+\frac{\nu_{3}(y)\left(x_{3}-y_{3}\right)}{|x-y|^{3}} e^{i \kappa|x-y|} \\
& -i \kappa \frac{\nu_{3}(y)\left(x_{3}+y_{3}\right)}{\left|x-y^{\prime}\right|^{2}} e^{i \kappa\left|x-y^{\prime}\right|}+\frac{\nu_{3}(y)\left(x_{3}+y_{3}\right)}{\left|x-y^{\prime}\right|^{3}} e^{i \kappa\left|x-y^{\prime}\right|}
\end{aligned}
$$

We proceed as in (3.6) and calculate

$$
\frac{e^{i \kappa|x-y|}}{|x-y|^{2}}=\frac{e^{i \kappa|\mathbf{x}-\mathbf{y}|}}{|\mathbf{x}-\mathbf{y}|^{2}}+\frac{i \kappa e^{i \kappa|\mathbf{x}-\mathbf{y}|}}{|\mathbf{x}-\mathbf{y}|^{3}} \frac{\left(x_{3}-y_{3}\right)^{2}}{2}+O\left(\frac{1}{|\mathbf{x}-\mathbf{y}|^{4}}\right) .
$$

We use this to transform (3.9) into

$$
\begin{aligned}
4 \pi \frac{\partial G\left(\mathbf{x}+h e_{3}, \mathbf{y}+s e_{3}\right)}{\partial \nu(y)}= & -\kappa^{2} \boldsymbol{\nu}(y) \cdot \frac{(\mathbf{x}-\mathbf{y})}{|\mathbf{x}-\mathbf{y}|} \frac{e^{i \kappa|\mathbf{x}-\mathbf{y}|}}{|\mathbf{x}-\mathbf{y}|^{2}} 2 h s \\
& -i \kappa \nu_{3}(y) \frac{e^{i \kappa|\mathbf{x}-\mathbf{y}|}}{|\mathbf{x}-\mathbf{y}|^{2}} 2 h+O\left(\frac{1}{|\mathbf{x}-\mathbf{y}|^{3}}\right),
\end{aligned}
$$

this equation holding in the same sense as (3.7).

4. Convolution and related integral operators. To establish that $S$ and $K$ are bounded operators on $L^{2}(\Gamma)$ and on $X$ we need tools from the theory of convolution operators and the Fourier and Hankel transforms. In this section we briefly recall the relevant results and compute explicitly certain Fourier transforms that we will need. The results in the first three paragraphs are contained, for example, in [23].

For $\ell \in L^{1}\left(\mathbb{R}^{2}\right) \cup L^{2}\left(\mathbb{R}^{2}\right)$ we define the Fourier transform of $\ell, \mathcal{F} \ell$, by

$$
(\mathcal{F} \ell)(\mathbf{k})=\frac{1}{2 \pi} \int_{\mathbb{R}^{2}} e^{-i \mathbf{k} \cdot \mathbf{y}} \ell(\mathbf{y}) d \mathbf{y}, \quad \mathbf{k} \in \mathbb{R}^{2} .
$$

In the case $\ell \in L^{1}\left(\mathbb{R}^{2}\right)$ the integral (4.1) exists in the ordinary Lebesgue sense, and $\mathcal{F} \ell \in B C\left(\mathbb{R}^{2}\right)$. If $\ell \in L^{2}\left(\mathbb{R}^{2}\right)$, then the integral (4.1) exists for almost all $\mathbf{k} \in \mathbb{R}^{2}$ as the limit

$$
\lim _{R \rightarrow \infty} \frac{1}{2 \pi} \int_{\mathbb{R}^{2} \cap B_{R}(0)} e^{-i \mathbf{k} \cdot \mathbf{y}} \ell(\mathbf{y}) d \mathbf{y}
$$


and $\mathcal{F} \ell \in L^{2}\left(\mathbb{R}^{2}\right)$, with $\|\mathcal{F} \ell\|_{L^{2}\left(\mathbb{R}^{2}\right)}=\|\ell\|_{L^{2}\left(\mathbb{R}^{2}\right)}$. Further, the mapping $\mathcal{F}: L^{2}\left(\mathbb{R}^{2}\right) \rightarrow$ $L^{2}\left(\mathbb{R}^{2}\right)$ is surjective and thus an isometric isomorphism. If $\ell \in L^{1}\left(\mathbb{R}^{2}\right) \cap L^{2}\left(\mathbb{R}^{2}\right)$, then the two interpretations of (4.1) coincide and $\mathcal{F} \ell \in X_{0}:=L^{2}\left(\mathbb{R}^{2}\right) \cap B C\left(\mathbb{R}^{2}\right)$.

For $\ell, \psi \in L^{2}\left(\mathbb{R}^{2}\right)$ we define $\ell * \psi$, the convolution of $\ell$ and $\psi$, by

$$
(\ell * \psi)(\mathbf{x}):=\int_{\mathbb{R}^{2}} \ell(\mathbf{x}-\mathbf{y}) \psi(\mathbf{y}) d \mathbf{y}, \quad \mathbf{x} \in \mathbb{R}^{2} .
$$

The integral is defined in the ordinary Lebesgue sense, and $\ell * \psi \in B C\left(\mathbb{R}^{2}\right)$. If also $\mathcal{F} \ell \in L^{\infty}\left(\mathbb{R}^{2}\right)$, then $\ell * \psi \in X_{0}$, with

$$
\ell * \psi=2 \pi \mathcal{F}^{-1}((\mathcal{F} \ell)(\mathcal{F} \psi))
$$

so that the convolution operator $L$, defined by $L \psi=\ell * \psi$, maps $L^{2}\left(\mathbb{R}^{2}\right)$ to $L^{2}\left(\mathbb{R}^{2}\right)$ and is bounded, with norm

$$
\left\|\left.L\right|_{L^{2}\left(\mathbb{R}^{2}\right) \rightarrow L^{2}\left(\mathbb{R}^{2}\right)} \leq 2 \pi\right\| \mathcal{F} \ell \|_{L^{\infty}\left(\mathbb{R}^{2}\right)} .
$$

We shall need in our arguments to also consider integral operators with kernels of a more general type. Suppose that $l: \mathbb{R}^{2} \times \mathbb{R}^{2} \rightarrow \mathbb{C}$ is such that $l(\mathbf{x}, \cdot)$ is measurable for all $\mathbf{x} \in \mathbb{R}^{2}$, and let $L$ be the integral operator with kernel $l$, so that

$$
(L \psi)(\mathbf{x})=\int_{\mathbb{R}^{2}} l(\mathbf{x}, \mathbf{y}) \psi(\mathbf{y}) d \mathbf{y}, \quad \mathbf{x} \in \mathbb{R}^{2} .
$$

One case of relevance to our later arguments is that in which

$$
l(\mathbf{x}, \mathbf{y})=m_{1}(\mathbf{x}) \ell(\mathbf{x}-\mathbf{y}) m_{2}(\mathbf{y}),
$$

with $m_{1}, m_{2} \in B C\left(\mathbb{R}^{2}\right), \ell \in L^{2}\left(\mathbb{R}^{2}\right), \mathcal{F} \ell \in L^{\infty}\left(\mathbb{R}^{2}\right)$. In this case, if $\psi \in L^{2}\left(\mathbb{R}^{2}\right)$, then (4.3) exists in the Lebesgue sense for all $\mathbf{x} \in \mathbb{R}^{2}, L \psi \in X_{0}$, and $L$ is a bounded operator on $L^{2}\left(\mathbb{R}^{2}\right)$ with norm

$$
\|L\|_{L^{2}\left(\mathbb{R}^{2}\right) \rightarrow L^{2}\left(\mathbb{R}^{2}\right)} \leq 2 \pi\left\|m_{1}\right\|_{B C\left(\mathbb{R}^{2}\right)}\|\mathcal{F} \ell\|_{L^{\infty}\left(\mathbb{R}^{2}\right)}\left\|m_{2}\right\|_{B C\left(\mathbb{R}^{2}\right)} .
$$

Clearly, $L$ is also a bounded operator on $L^{2}\left(\mathbb{R}^{2}\right)$ if it is a sum of operators of this form.

Another case of relevance is that in which

$$
|l(\mathbf{x}, \mathbf{y})| \leq \ell(\mathbf{x}-\mathbf{y}),
$$

with $\ell \in L^{p}\left(\mathbb{R}^{2}\right)$, for some $p \in[1, \infty)$. In this case, if $\psi$ is continuous and compactly supported, then (4.3) exists in the Lebesgue sense for all $\mathbf{x} \in \mathbb{R}^{2}, L \psi \in L^{s}\left(\mathbb{R}^{2}\right)$, for $s \geq 1$, and, from Young's inequality [23], it follows that

$$
\|L \psi\|_{L^{s}\left(\mathbb{R}^{2}\right)} \leq\|\ell\|_{L^{p}\left(\mathbb{R}^{2}\right)}\|\psi\|_{L^{r}\left(\mathbb{R}^{2}\right)},
$$

where $r^{-1}=1+s^{-1}-p^{-1}$. Since the set of continuous compactly supported functions is dense in $L^{r}\left(\mathbb{R}^{2}\right)$, we can extend the domain of $L$ by density so that $L$ is a bounded operator from $L^{r}\left(\mathbb{R}^{2}\right)$ to $L^{s}\left(\mathbb{R}^{2}\right)$ with norm $\leq\|\ell\|_{L^{p}\left(\mathbb{R}^{2}\right)}$. Further, if $\ell \in L^{1}\left(\mathbb{R}^{2}\right)$ and $\psi \in L^{\infty}\left(\mathbb{R}^{2}\right)$, then, trivially, (4.3) exists in the Lebesgue sense for all $\mathbf{x} \in \mathbb{R}^{2}$ and (4.7) holds. 
We will use the bound (4.7) particularly often in the case $\ell \in L^{1}\left(\mathbb{R}^{2}\right)$, in which case it implies that

$$
\|L\|_{L^{q}\left(\mathbb{R}^{2}\right) \rightarrow L^{q}\left(\mathbb{R}^{2}\right)} \leq\|\ell\|_{L^{1}\left(\mathbb{R}^{2}\right)}
$$

for $1 \leq q \leq \infty$. A further consequence of (4.7) is the following result that will be used to prove Lemma 5.1.

Lemma 4.1. Suppose that $L$ is the integral operator given by (4.3), and that the bound (4.6) holds with $\ell \in L^{1}\left(\mathbb{R}^{2}\right) \cap L^{p}\left(\mathbb{R}^{2}\right)$, for some $p \in(1,2)$. Then, for some $n \in \mathbb{N}, L^{n}$ is a bounded operator from $L^{2}\left(\mathbb{R}^{2}\right)$ to $L^{\infty}\left(\mathbb{R}^{2}\right)$.

Proof. Note first that $\ell \in L^{1}\left(\mathbb{R}^{2}\right) \cap L^{p}\left(\mathbb{R}^{2}\right)$ implies that $\ell \in L^{\tilde{p}}\left(\mathbb{R}^{2}\right)$ for $1<\tilde{p}<p$. Let $\omega:=p /(p-1)>2$. Define the finite or infinite sequence $\left(r_{j}\right)$ iteratively by

$$
r_{0}:=2, \quad r_{j+1}:=\left(\frac{1}{p}+\frac{1}{r_{j}}-1\right)^{-1}=\frac{r_{j}}{1-\frac{r_{j}}{\omega}}, j=0,1,2, \ldots,
$$

continuing the definition (4.9) for as long as $r_{j}<\omega$. Let $J \subset \mathbb{N}_{0}:=\mathbb{N} \cup\{0\}$ denote the set of indices $j$ for which $r_{j}$ is defined. We will show that the set $J$ is finite, so that $J=\{0,1, \ldots, N\}$ with $r_{N} \geq \omega$. Then, by (4.7), it follows that $L$ is a bounded operator from $L^{r_{j-1}}\left(\mathbb{R}^{2}\right)$ to $L^{r_{j}}\left(\mathbb{R}^{2}\right)$ for $j=1, \ldots, N$. Further, defining $\tilde{p}:=r_{N} /\left(r_{N}-1\right)$, we observe that $1<\tilde{p}<p$ and $\frac{1}{\tilde{p}}+\frac{1}{r_{N}}=1$, so that, by (4.7), $L$ is a bounded operator from $L^{r_{N}}\left(\mathbb{R}^{2}\right)$ to $L^{\infty}\left(\mathbb{R}^{2}\right)$, and so $L^{N+1}$ is a bounded operator from $L^{2}\left(\mathbb{R}^{2}\right)$ to $L^{\infty}\left(\mathbb{R}^{2}\right)$.

We complete the proof by showing that $J$ is finite. Suppose otherwise. Then $r_{j}<\omega$ for all $j \in J=\mathbb{N}_{0}$. It follows from (4.9), by induction, that the sequence $\left(r_{j}\right)$ is monotonically increasing. Thus the sequence $\left(r_{j}\right)$ is convergent to some limit $r$, with $2 \leq r \leq \omega$. Rearranging (4.9) and taking limits, we see that $(1-r / \omega) r=r$, so that $r=0$, a contradiction.

Examining (3.7) and (3.9) we see that large parts of the kernels of the operators $S$ and $K$ have the form (4.4), where, moreover, $\ell$ has certain symmetries that simplify the calculation of its Fourier transform. For the remainder of this section, for $\mathbf{y} \in \mathbb{R}^{2}$ let $r:=|\mathbf{y}|$ and $\hat{\mathbf{y}}:=\mathbf{y} /|\mathbf{y}|$. The specific symmetries that arise are those where $\ell$ has the form

$$
\ell(\mathbf{y})=F(r) Y_{n}^{j}(\hat{\mathbf{y}})
$$

with $n=0$ or 1 , and $j=0, \ldots, n$, where the functions $Y_{n}^{j}$ are spherical harmonics of order $n$ defined on the unit circle $\Omega \subset \mathbb{R}^{2}$ by

$$
Y_{0}^{0}(\hat{\mathbf{y}}):=1, \quad Y_{1}^{0}(\hat{\mathbf{y}}):=\cos \theta, \quad Y_{1}^{1}(\hat{\mathbf{y}}):=\sin \theta, \quad \hat{\mathbf{y}}=(\cos \theta, \sin \theta) \in \Omega .
$$

Integrating the product of the Jacobi-Anger expansion [13, equation (3.66)] (a Fourier series of $\left.e^{i r \mathbf{k} \cdot \hat{\mathbf{y}}}\right)$ with the spherical harmonics of order $n$ over the unit circle we deduce the Funk-Hecke formulae in $\mathbb{R}^{2}$,

$$
\int_{\Omega} e^{-i r \mathbf{k} \cdot \hat{\mathbf{y}}} Y_{n}^{j}(\hat{\mathbf{y}}) d s(\hat{\mathbf{y}})=2 \pi i^{n} J_{n}(r k) Y_{n}^{j}(\hat{\mathbf{k}}),
$$

where we define $k:=|\mathbf{k}|$ and $\hat{\mathbf{k}}:=\mathbf{k} / k$ and $J_{n}$ denotes the Bessel function of order $n$. 
If $\ell \in L^{1}\left(\mathbb{R}^{2}\right) \cup L^{2}\left(\mathbb{R}^{2}\right)$ has the form (4.10), then it holds for almost all $\mathbf{k} \in \mathbb{R}^{2}$ that

$$
\begin{aligned}
(\mathcal{F} \ell)(\mathbf{k}) & =\lim _{R \rightarrow \infty} \frac{1}{2 \pi} \int_{|\mathbf{y}|<R} e^{-i \mathbf{k} \cdot \mathbf{y}} \ell(\mathbf{y}) d \mathbf{y} \\
& =\frac{1}{2 \pi} \lim _{R \rightarrow \infty} \int_{0}^{R}\left(\int_{\Omega} e^{-i r \mathbf{k} \cdot \hat{\mathbf{y}}} Y_{n}^{j}(\hat{\mathbf{y}}) d s(\hat{\mathbf{y}})\right) F(r) r d r \\
& =i^{n} Y_{n}^{j}(\hat{\mathbf{k}}) \lim _{R \rightarrow \infty} \int_{0}^{R} F(r) J_{n}(k r) r d r \\
& =\frac{i^{n} Y_{n}^{j}(\hat{\mathbf{k}})}{\sqrt{k}} \mathcal{H}_{n}(\sqrt{\cdot} \cdot F(\cdot))(k),
\end{aligned}
$$

where $\mathcal{H}_{n}$ denotes the Hankel transform of order $n$, i.e.,

$$
\left(\mathcal{H}_{n} F\right)(k):=\lim _{R \rightarrow \infty} \int_{0}^{R} F(r) J_{n}(k r) \sqrt{k r} d r, \quad k \in \mathbb{R}^{+}, \quad n \in \mathbb{N} .
$$

We note that (4.13) can be used to extend the domain of the Fourier transform. Precisely, whenever $\ell$ has the form (4.10) and the limit (4.13) exists, (4.13) can be used to define a Fourier transform of $\ell$, this definition coinciding with the usual one if $\ell \in L^{1}\left(\mathbb{R}^{2}\right) \cup L^{2}\left(\mathbb{R}^{2}\right)$. An example is the function $\ell$ defined, for some $h>0$, by

$$
\ell(\mathbf{y}):=W_{h}(|\mathbf{y}|), \quad W_{h}(r):=\frac{1}{4 \pi} \frac{e^{i \kappa \sqrt{r^{2}+4 h^{2}}}}{\sqrt{r^{2}+4 h^{2}}}, \quad r>0 .
$$

The relevance of this example is that, defining $x_{h}:=(0,0,2 h), \ell=\Phi\left(\cdot, x_{h}\right)$ is the trace of $\Phi\left(\cdot, x_{h}\right)$ on the plane $\Gamma_{0}$. It is not difficult to see, from the asymptotic behavior of the Bessel function $J_{n}$ in (4.20), that, for $F=W_{h}$, the limit (4.13) is well defined except for $k=\kappa$ in the case $\kappa>0$. Explicitly, from (4.14) with $n=0$ and the Hankel transforms in section 8.2 of [18], namely formula (24) for $\kappa_{1}>0$ and formulae (41) and (50) for $\kappa_{1}=0$, we find after some elementary calculations that, for all $\mathbf{k} \in \mathbb{R}^{2}$ with $k=|\mathbf{k}| \neq \kappa$,

$$
(\mathcal{F} \ell)(\mathbf{k})=\left(\mathcal{F} \Phi\left(\cdot, x_{h}\right)\right)(\mathbf{k})=\int_{0}^{\infty} W_{h}(r) J_{0}(k r) r d r=\frac{1}{4 \pi} \frac{e^{-2 h \sqrt{k^{2}-\kappa^{2}}}}{\sqrt{k^{2}-\kappa^{2}}},
$$

where the square root is chosen so that its argument lies in $[-\pi / 2,0]$.

We now use the representation (4.13) to calculate the Fourier transforms of parts of the kernels of the operators $S$ and $K$. We suppose that $\ell$ is given by (4.10), with $n=0$ or 1 and

$$
F(r):=\frac{e^{i \kappa r}}{\beta+r^{2}}, \quad r \geq 0
$$

for some $\beta>0$. The relevance of this example to the operators $S$ and $K$ is that the explicitly written terms on the right-hand side of (3.7) and (3.9) all take the form (4.4) if $\ell$ is given by (4.10) and (4.18) with $\beta=0$ and if $\mathbf{x}+h e_{3}$ and $\mathbf{y}+s e_{3}$ lie on $\Gamma$.

Clearly, $\ell \in L^{2}\left(\mathbb{R}^{2}\right)$ (for $\beta>0$ ). We will show also that the Fourier transform of $\ell$ is bounded, so that the operation of convolution with $\ell$ is bounded on $L^{2}\left(\mathbb{R}^{2}\right)$. To this end we show that the improper integral

$$
I(k):=\int_{0}^{\infty} F(r) J_{n}(k r) r d r
$$

is bounded on $[0, \infty)$. 
With the help of the asymptotic expansion of the Bessel function (see, e.g., [1]),

$$
J_{n}(z)=\sqrt{\frac{2}{\pi z}} \cos \left(z-\frac{n \pi}{2}-\frac{\pi}{4}\right)\left\{1+O\left(\frac{1}{z}\right)\right\}, \quad|z| \rightarrow \infty,
$$

we see that $e^{i z} J_{n}(z)$ is bounded in $0 \leq \arg z \leq \theta$ for every $\theta \in(0, \pi / 2)$. Since $\operatorname{Re}(i(\kappa-k) z)=-\left(\kappa_{0}-k\right) \operatorname{Im} z-\kappa_{1} \operatorname{Re} z$ and $F(z)$ is a holomorphic function in $\operatorname{Re} z>0$, we see that for $0 \leq k<\kappa_{0}$ we may transform the integral

$$
I(k)=\int_{0}^{\infty} \frac{e^{i(\kappa-k) z}}{\beta+z^{2}} e^{i k z} J_{n}(k z) z d z
$$

into

$$
I(k)=\int_{\gamma} \frac{e^{i(\kappa-k) z}}{\beta+z^{2}} e^{i k z} J_{n}(k z) z d z
$$

with $\gamma=\{(1+i) t: t \geq 0\}$. This integral is bounded for $0 \leq k \leq \kappa_{0} / 2$.

For $k \geq \kappa_{0} / 2$ we can use (4.20) and that $J_{n}(z)$ is continuous and thus, by (4.20), bounded on $[0, \infty)$ to estimate that, for some constants $C_{1}$ and $C_{2}$,

$$
\left|\int_{0}^{\infty} F(r) J_{n}(k r) r d r\right| \leq C_{1}+C_{2} \int_{1}^{\infty} \frac{1}{r^{3 / 2}} d r .
$$

We conclude that $I$ is bounded on $[0, \infty)$ for $n=0,1$, so that, by (4.13), $\mathcal{F} \ell \in L^{\infty}\left(\mathbb{R}^{2}\right)$ for $n=0,1$.

We will be interested in the last section of the paper, in order to establish a limiting absorption principle, in the dependence of $\ell$ on $\kappa_{1}$. Denote, temporarily, $\ell$ and $I$ by $\ell_{\kappa_{1}}$ and $I_{\kappa_{1}}$ to indicate their dependence on $\kappa_{1}$. Then, from (4.22), since $e^{i z} J_{n}(z)$ is bounded on $\gamma=\{(1+i) t: t \geq 0\}$, we see that, for some constant $C>0$,

$$
\left|I_{\kappa_{1}}(k)-I_{0}(k)\right| \leq C \int_{0}^{\infty} e^{-\kappa_{0} t / 2}\left(1-e^{-\kappa_{1} t}\right) d t
$$

for $0 \leq k \leq \kappa_{0} / 2, \kappa_{1} \geq 0$, so that $I_{\kappa_{1}}(k) \rightarrow I_{0}(k)$ as $\kappa_{1} \rightarrow 0$, uniformly on $\left[0, \kappa_{0} / 2\right]$. Similarly, using (4.21) and (4.20) (cf. (4.23)), we can show that $I_{\kappa_{1}}(k) \rightarrow I_{0}(k)$ as $\kappa_{1} \rightarrow 0$, uniformly on $\left[\kappa_{0} / 2, \infty\right]$. Thus the following lemma holds.

LEMMA 4.2. If $\ell$ is given by (4.10) and (4.18) with $\beta>0$ and $n=j=0$, or $n=1$ and $j=0$, or 1 , then $\mathcal{F} \ell \in L^{\infty}\left(\mathbb{R}^{2}\right)$ so that the convolution integral operator $L$, with kernel $\ell(\mathbf{x}-\mathbf{y})$, is a bounded operator on $L^{2}\left(\mathbb{R}^{2}\right)$. Further, denoting $\ell$ and $L$ by $\ell_{\kappa_{1}}$ and $L_{\kappa_{1}}$ to indicate their dependence on $\kappa_{1}$, we have that $\left\|\mathcal{F} \ell_{\kappa_{1}}-\mathcal{F} \ell_{0}\right\|_{L^{\infty}\left(\mathbb{R}^{2}\right)} \rightarrow 0$ as $\kappa_{1} \rightarrow 0$, so that $L_{\kappa_{1}}$ tends to $L_{0}$ in norm as $\kappa_{1} \rightarrow 0$.

5. Properties of single- and double-layer potentials. In this section we prove that the single- and double-layer operators are well defined when considered as operators on $L^{2}(\Gamma)$. We further investigate the jump relations for unbounded regions and show continuity properties of the boundary operators with respect to variations of the boundary.

We first prove Theorem 2.1, stated at the end of section 2. To prove this result we split the operators into a local and a global part with the help of an appropriate cut-off function. To this end let $\chi:[0, \infty) \rightarrow \mathbb{R}$ be a continuous function with

$$
\chi(t):=\left\{\begin{array}{ll}
0, & t<1 / 2, \\
1, & t \geq 1,
\end{array} \quad \text { and } \quad 0 \leq \chi(t) \leq 1 \quad \forall t \geq 0 .\right.
$$


Let $A$ with kernel $a$ denote one of the operators $S$ or $K$, respectively. We define the global part

$$
\left(A_{1} \varphi\right)(x):=\int_{\Gamma} \chi(|x-y|) a(x, y) \varphi(y) d s(y), \quad x \in \Gamma,
$$

and the local part

$$
\left(A_{2} \varphi\right)(x):=\int_{\Gamma}(1-\chi(|x-y|)) a(x, y) \varphi(y) d s(y), \quad x \in \Gamma .
$$

This yields the decomposition $A=A_{1}+A_{2}$, and we can study the mapping properties of $A_{1}$ and $A_{2}$ as operators on $L^{2}(\Gamma)$ and on $X$ separately. We start by proving the following lemma.

Lemma 5.1. $A_{2}$ is a bounded operator on $L^{q}(\Gamma)$ for $1 \leq q \leq \infty$, is a bounded operator from $L^{\infty}(\Gamma)$ to $B C(\Gamma)$, and is a bounded operator on $X$. Further, for some $n \in \mathbb{N}, A_{2}^{n}$ is a bounded operator from $L^{2}(\Gamma)$ to $X$.

Proof. The kernel $a_{2}$ of $A_{2}$ has compact support and is weakly singular. Precisely, since (cf. [7, equation (4.23)])

$$
|\nu(y) \cdot(x-y)| \leq|\mathbf{x}-\mathbf{y}|^{1+\alpha}|| f \|_{B C^{1, \alpha}\left(\mathbb{R}^{2}\right)}, \quad x, y \in \Gamma,
$$

it holds in the double-layer case $A=K$ that, for some constant $C>0$,

$$
\left|a_{2}(x, y)\right| \leq C \ell(\mathbf{x}-\mathbf{y}), \quad x, y \in \Gamma, x \neq y,
$$

where

$$
\ell(\mathbf{y}):=\left\{\begin{array}{cc}
|\mathbf{y}|^{\alpha-2}, & |\mathbf{y}| \leq 1 \\
0, & |\mathbf{y}|>1
\end{array}\right.
$$

The same bound holds (but is not sharp) in the single-layer case $A=S$.

Since $\ell \in L^{p}\left(\mathbb{R}^{2}\right)$, for $1 \leq p<2 /(2-\alpha)$, we see from (4.8) that $A_{2}$ is a bounded operator on $L^{q}(\Gamma)$ for $1 \leq q \leq \infty$. Since $a_{2}(x, y)$ is also continuous for $x \neq y$, it follows, moreover, that $A_{2}$ maps $L^{\infty}(\Gamma)$ to $B C(\Gamma)$. Thus $A_{2}$ is a bounded operator on $X$. By Lemma 4.1, $A_{2}^{m}$ is a bounded operator from $L^{2}(\Gamma)$ to $L^{\infty}(\Gamma)$, for some $m \in \mathbb{N}$, so that $A_{2}^{m+1}$ is a bounded operator from $L^{2}(\Gamma)$ to $X$.

We now consider the global part and prove a lemma on the mapping properties of $A_{1}$. Together, Lemmas 5.1 and 5.2 provide a proof of Theorem 2.1.

LEMma 5.2. $A_{1}$ is a bounded operator on $L^{2}(\Gamma)$ and is a bounded operator from $L^{2}(\Gamma)$ to $X$.

Proof. From the decompositions (3.7) and (3.11) it follows that the kernel $a_{1}$ of $A_{1}$ can be written, in both the cases $A=S$ and $A=K$, in the form

$$
a_{1}(x, y)=l^{*}(\mathbf{x}, \mathbf{y})+l(\mathbf{x}, \mathbf{y}),
$$

where $l^{*}$ is a sum of terms each of the form (4.4), with $m_{1}, m_{2} \in B C\left(\mathbb{R}^{2}\right)$ and $\ell$ given by (4.10) and (4.18) with $\beta=1$, and with $n=0$ or 1 . Further, $l^{*}$ can be chosen so that $l$ satisfies the bound, for some constant $C>0$,

$$
|l(\mathbf{x}, \mathbf{y})| \leq C \tilde{\ell}(\mathbf{x}-\mathbf{y}), \quad \mathbf{x}, \mathbf{y} \in \mathbb{R}^{2},
$$


where $\tilde{\ell}(\mathbf{y}):=(1+|\mathbf{y}|)^{-3}$, so that $\tilde{\ell} \in L^{1}\left(\mathbb{R}^{2}\right)$. In detail, in the case $A=S$ we see from (3.7) that an appropriate choice is to take

$$
l^{*}(\mathbf{x}, \mathbf{y})=-\frac{i \kappa f(\mathbf{x}) f(\mathbf{y})}{2 \pi} \frac{e^{i \kappa|\mathbf{x}-\mathbf{y}|}}{1+|\mathbf{x}-\mathbf{y}|^{2}},
$$

while, in the case $A=K$ we see from (3.11) that we can take

$$
l^{*}(\mathbf{x}, \mathbf{y})=-\frac{\kappa^{2} f(\mathbf{x}) f(\mathbf{y})}{2 \pi} \boldsymbol{\nu}(y) \cdot \frac{\mathbf{x}-\mathbf{y}}{|\mathbf{x}-\mathbf{y}|} \frac{e^{i \kappa|\mathbf{x}-\mathbf{y}|}}{1+|\mathbf{x}-\mathbf{y}|^{2}}-\frac{i \kappa f(\mathbf{x}) \nu_{3}(y)}{2 \pi} \frac{e^{i \kappa|\mathbf{x}-\mathbf{y}|}}{1+|\mathbf{x}-\mathbf{y}|^{2}} .
$$

It follows from (4.5) and Lemma 4.2 applied to the integral operator with kernel $l^{*}$, and (4.8) applied to the integral operator with kernel $l$, that $A_{1}$ is a bounded operator on $L^{2}(\Gamma)$.

Note, from the above representation (5.7), that also

$$
\left|a_{1}(x, y)\right| \leq \ell^{*}(\mathbf{x}-\mathbf{y}), \quad x, y \in \Gamma,
$$

for some $\ell^{*} \in L^{2}\left(\mathbb{R}^{2}\right)$; this is true since $\tilde{\ell} \in L^{2}\left(\mathbb{R}^{2}\right)$ and since each term of (5.9) and (5.10) can be bounded in this way. It follows from (4.7) that $A_{1}$ maps $L^{2}(\Gamma)$ to $L^{\infty}(\Gamma)$; in fact, since also $a_{1}$ is continuous, it holds that $A_{1}$ maps $L^{2}(\Gamma)$ to $B C(\Gamma)$.

Remark 5.3. For $C_{2}>C_{1}>0$ let

$$
B=B\left(C_{1}, C_{2}\right):=\left\{f \in B C^{1, \alpha}\left(\mathbb{R}^{2}\right): C_{1} \leq f(\mathbf{y}), \mathbf{y} \in \mathbb{R}^{2}, \quad\|f\|_{B C^{1, \alpha}\left(\mathbb{R}^{2}\right)} \leq C_{2}\right\} .
$$

We note that, given $C_{2}>C_{1}>0$ and $\kappa_{0}>0$, we can choose $C>0$ such that the estimates (5.5) and (5.8) hold for all $f \in B$ and all $\kappa_{1} \geq 0$. (For (5.8) this follows from (3.7) and (3.11).) This observation will be helpful in establishing continuous dependence of $A$ on $f$ and on $\kappa_{1}$.

Combining the above lemmas we deduce Theorem 2.1 and have also the following corollary.

CoROLlary 5.4. For all sufficiently large $n \in \mathbb{N}$ it holds that $A^{n}$ is a bounded map from $L^{2}(\Gamma)$ to $X$.

As part of the proof of Theorem 2.2 we need to show that our modified single- and double-layer potentials $u_{1}$ and $u_{2}$, over the unbounded surface $\Gamma$, behave in a similar way to the corresponding standard layer potentials supported on a smooth bounded surface. This is done in the following theorem, in which $M:=\left\{x: 0<x_{3}<f(\mathbf{x})\right\}$ denotes the region between $\Gamma$ and $\Gamma_{0}$.

TheOREM 5.5. Let $u_{1}$ and $u_{2}$ denote the single- and double-layer potentials with density $\varphi \in X$, defined by (2.14) and (2.15), respectively. The following hold:

(i) For $n=1,2, u_{n} \in C^{2}(D \cup M)$ and $\Delta u_{n}+k^{2} u_{n}=0$ in $D \cup M$.

(ii) $u_{1}$ and $u_{2}$ can be continuously extended from $D$ to $\bar{D}$ and from $M$ to $\bar{M}$, with limiting values

$$
u_{1, \pm}(x)=\int_{\Gamma} G(x, y) \varphi(y) d s(y), \quad x \in \Gamma
$$

and

$$
u_{2, \pm}(x)=\int_{\Gamma} \frac{\partial G(x, y)}{\partial \nu(y)} \varphi(y) d s(y) \pm \frac{1}{2} \varphi(x), \quad x \in \Gamma
$$


where $u_{n, \pm}(x):=\lim _{\epsilon \rightarrow 0+} u_{n}(x \pm \epsilon \nu(x))$, for $n=1,2$ and $x \in \Gamma$, and $\nu(x)$ denotes the unit normal at $x \in \Gamma$ directed into $D$.

(iii) Given constants $C_{2}>C_{1}>0$ and a compact subset $S$ of $H^{+}$, there exists a constant $C>0$ such that

$$
\left|u_{n}(x)\right| \leq C\|\varphi\|_{X}, \quad x \in D \cup M, n=1,2,
$$

for all $\varphi \in X, \kappa \in S$, and $f \in B=B\left(C_{1}, C_{2}\right)$.

(iv) Given constants $C_{2}>C_{1}>0$ and $\epsilon>0$ and a compact subset $S$ of $H^{+}$, there exists a constant $C>0$ such that

$$
\left|u_{n}(x)\right| \leq C\|\varphi\|_{L^{2}(\Gamma)}, \quad n=1,2,
$$

for all $x \in D \cup M$ with $\left|x_{3}-f\left(x_{1}, x_{2}\right)\right|>\epsilon$, all $\varphi \in X$, all $\kappa \in S$, and all $f \in B=$ $B\left(C_{1}, C_{2}\right)$.

Proof. We first show that $u \in C(D \cup M)$ and establish (ii) and (iii). We use the cut-off function $\chi$ given by (5.1). Let $u$ denote one of $u_{1}$ and $u_{2}$, and let $a$ denote the kernel of $u$ so that $a(x, y):=G(x, y)$ and $a(x, y):=\partial G(x, y) / \partial \nu(y)$ in the respective cases. We have, for $x \in D \cup M$, that

$$
u(x)=\int_{\Gamma} \chi(|x-y|) a(x, y) \varphi(y) d s(y)+\int_{\Gamma}[1-\chi(|x-y|)] a(x, y) \varphi(y) d s(y) .
$$

The first term has a continuous kernel that is bounded at infinity by the estimate (3.7) or (3.9) and, since $\varphi \in L^{2}(\Gamma)$, is continuous in $\left\{x: x_{3}>0\right\}$. The second term is clearly continuous in $D \cup M$; to see that it can be continuously extended up to $\Gamma$ from above and below and to compute its limiting values we observe that, keeping $x$ within some ball centered at some $x_{0} \in \Gamma$, it holds that the integrand is supported in a finite patch of the surface. We can extend this surface patch to a bounded obstacle with boundary of class $C^{1, \alpha}$ and, since $\varphi \in C(\Gamma)$, use the jump relations for bounded obstacles as presented in [14].

To show that the first term satisfies the bound (5.14) we recall that $G$ satisfies the bound (3.8) and point out that, by interior elliptic regularity estimates for solutions of the Helmholtz equation (e.g., [9, Lemma 2.7]), it follows that $\nabla_{y} G(x, y)$ satisfies the same bound for all $\kappa \in S$ (with a different constant $C$ ), provided $x_{3}, y_{3}>0$ and $|x-y|>1 / 4$; further we calculate directly that $C$ can be chosen so that this bound also holds for $0<|x-y| \leq 1 / 4$. Thus, for some constant $C^{\prime}>0$, whether $a$ is the kernel of the single- or double-layer potential, it holds for all $\kappa \in S$ that

$$
|\chi(|x-y|) a(x, y)| \leq C^{\prime} \frac{\left(1+x_{3}\right)\left(1+y_{3}\right)}{1+|x-y|^{2}}, \quad x, y \in \mathbb{R}^{3}, x_{3}, y_{3} \geq 0 .
$$

Applying the Cauchy-Schwarz inequality we have that the first term is bounded, for $x \in\left\{x: x_{3}>0\right\}$, by $C^{\prime}\left(1+f_{+}\right) I(x)\|\varphi\|_{L^{2}(\Gamma)}$, where

$$
\begin{aligned}
{[I(x)]^{2} } & =\left(1+x_{3}\right)^{2} \int_{\Gamma} \frac{d s(y)}{\left(1+|x-y|^{2}\right)^{2}} \\
& \leq\left(1+x_{3}\right)^{2}\left(1+\|\nabla f\|_{B C(\Gamma)}\right)^{1 / 2} \int_{\mathbb{R}^{2}} \frac{d \mathbf{y}}{\left(1+|\mathbf{x}-\mathbf{y}|^{2}+\left(x_{3}-f(\mathbf{y})\right)^{2}\right)^{2}} .
\end{aligned}
$$

Thus, for some constant $c>0$ it holds, for all $x \in\left\{y: y_{3}>0\right\}$ and all $f \in B$, that $[I(x)]^{2} \leq c F\left(x_{3}\right)$, where

$$
F\left(x_{3}\right):=\left(1+x_{3}\right)^{2} \int_{0}^{\infty} \frac{r d r}{\left(1+x_{3}^{2}+r^{2}\right)^{2}}=\frac{\left(1+x_{3}\right)^{2}}{x_{3}^{2}} \int_{0}^{\infty} \frac{s d s}{\left(x_{3}^{-2}+1+s^{2}\right)^{2}} .
$$

Clearly, $F$ is bounded on $[0, \infty)$. Thus the first term satisfies the bound (5.14). 
To treat the second term we argue analogously to the corresponding 2D case [7]. We remark that $1-\chi(|x-y|)$ is zero for $|x-y| \geq 1$. We consider only the double-layer case $u=u_{1}$. (The argument is similar but simpler in the single-layer case.) Directly from the definitions (see (3.9)) we see that there exists a constant $C>0$ such that

$$
|(1-\chi(|x-y|)) a(x, y)| \leq C \frac{|\nu(y) \cdot(x-y)|}{|x-y|^{3}}, \quad x \in D \cup M, y \in \Gamma,
$$

for all $\kappa \in S$ and all $f \in B$. Define $x^{*}:=(\mathbf{x}, f(\mathbf{x})) \in \Gamma, \delta:=\left|f(\mathbf{x})-x_{3}\right|$, and note that, by the triangle inequality,

$$
\left(|\mathbf{x}-\mathbf{y}|^{2}+\delta^{2}\right)^{1 / 2} \leq|x-y|+|f(\mathbf{x})-f(\mathbf{y})| \leq\left(1+\|\nabla f\|_{B C(\Gamma)}\right)|x-y| .
$$

Using this inequality, and (5.4) to bound $\left|\nu(y) \cdot\left(x^{*}-y\right)\right|$, we see that, for some $C^{\prime}>0$,

$$
|(1-\chi(|x-y|)) a(x, y)| \leq C^{\prime} \frac{|\mathbf{x}-\mathbf{y}|^{1+\alpha}+\delta}{\left(|\mathbf{x}-\mathbf{y}|^{2}+\delta^{2}\right)^{3 / 2}}, \quad x \in D \cup M, y \in \Gamma,
$$

for all $\kappa \in S$ and $f \in B$. Thus, defining $C^{\prime \prime}=C^{\prime}\left(1+\|\nabla f\|_{B C(\Gamma)}\right)^{1 / 2}$, the second term is bounded by

$$
C^{\prime \prime}\|\varphi\|_{B C(\Gamma)} \int_{|\mathbf{y}|<1} \frac{|\mathbf{y}|^{1+\alpha}+\delta}{\left(|\mathbf{y}|^{2}+\delta^{2}\right)^{3 / 2}} d \mathbf{y} \leq 2 \pi C^{\prime \prime}\|\varphi\|_{B C(\Gamma)} \int_{0}^{1} \frac{r^{1+\alpha}+\delta}{r^{2}+\delta^{2}} d r,
$$

for all $\kappa \in S$ and $f \in B$, so that the second term satisfies the bound (5.14).

To establish (iv) we modify the argument used to show (iii). We have remarked above that both $G(x, y)$ and $\nabla_{y} G(x, y)$ satisfy the bound (3.8). Thus (cf. (5.16)), for every $\epsilon>0$ there exists $C_{\epsilon}>0$ such that

$$
|a(x, y)| \leq C_{\epsilon} \frac{\left(1+x_{3}\right)\left(1+y_{3}\right)}{1+|x-y|^{2}}
$$

for all $x, y \in \mathbb{R}^{3}$ with $x_{3}, y_{3} \geq 0$ and $|x-y| \geq \epsilon$ and all $\kappa \in S$. Applying the CauchySchwarz inequality, as in the proof of (5.14), we see that it holds, for some constant $C_{\epsilon}^{\prime}>0$, that

$$
\left|u_{n}(x)\right| \leq C_{\epsilon}^{\prime}\left(1+f_{+}\right) I(x)\|\varphi\|_{L^{2}(\Gamma)}, \quad n=1,2,
$$

for all $x \in D \cup M$ with $\left|x_{3}-f\left(x_{1}, x_{2}\right)\right| \geq \epsilon$ and all $\kappa \in S$ and $f \in B$. In view of the bound on $I(x)$ already shown above, we see that we have established (5.15).

We complete the proof by establishing (i). This is clear when $\varphi$ is compactly supported. The general case follows from the density in $L^{2}(\Gamma)$ of the set of those elements of $X$ that are compactly supported, from the bound (5.15), and from the fact that limits of uniformly convergent sequences of solutions of the Helmholtz equation satisfy the Helmholtz equation (e.g., [9, Remark 2.8]).

We continue this section by proving that the single- and double-layer potential operators depend continuously on variations in the boundary $\Gamma$. In the statement of the following theorem, $B=B\left(C_{1}, C_{2}\right)$ is the set defined in Remark 5.3 for some constants $C_{2}>C_{1}>0$. We use the notation $A_{f}$ for either $S$ or $K$ defined on a surface $\Gamma_{f}$ given by some $f \in B$. With the help of the isomorphism

$$
I_{f}: L^{2}\left(\Gamma_{f}\right) \rightarrow L^{2}\left(\mathbb{R}^{2}\right), \quad\left(I_{f} \varphi\right)(\mathbf{y})=\varphi((\mathbf{y}, f(\mathbf{y}))), \quad \mathbf{y} \in \mathbb{R}^{2},
$$


we associate $A_{f}$ with the element $\tilde{A}_{f}=I_{f} A_{f} I_{f}^{-1}$ of the set of bounded linear operators on $L^{2}\left(\mathbb{R}^{2}\right)$ for each $f \in B$. Denoting the kernel of $\tilde{A}_{f}$ by $a_{f}$, we see that, where $x=(\mathbf{x}, f(\mathbf{x})), y=(\mathbf{y}, f(\mathbf{y}))$, and $a(x, y):=G(x, y)$ or $a(x, y):=\partial G(x, y) / \partial \nu(y)$, in the respective cases $A_{f}=S$ and $A_{f}=K$, it holds that

$$
a_{f}(\mathbf{x}, \mathbf{y})=a(x, y) J_{f}(\mathbf{y}), \quad J_{f}(\mathbf{y}):=\sqrt{1+|\nabla f(\mathbf{y})|^{2}} .
$$

THEOREM 5.6. The single- and double-layer potential operators depend continuously on the boundary $\Gamma_{f}$ of the unbounded domain $D_{f}$ in the sense that

$$
\sup _{\substack{f, g \in B \\ g \|_{B C^{1, \alpha}\left(\mathbb{R}^{2}\right)} \leq \epsilon}}\left\|\tilde{A}_{f}-\tilde{A}_{g}\right\|_{L^{2}\left(\mathbb{R}^{2}\right) \rightarrow L^{2}\left(\mathbb{R}^{2}\right)} \rightarrow 0, \quad \epsilon \rightarrow 0 .
$$

Proof. Similarly to how we proceeded when proving Theorem 2.1, we decompose the operator $\tilde{A}_{f}-\tilde{A}_{g}$ into a global and a local part, i.e., $\tilde{A}_{f}-\tilde{A}_{g}=A_{1}+A_{2}$ with $A_{1}, A_{2}$ defined similarly to (5.2) and (5.3). We now carry out the proof for the case of the single-layer operator. The necessary changes for the double-layer operator are straightforward.

The global operator. The kernel of the global operator $A_{1}$ is given by

$$
a_{1}(\mathbf{x}, \mathbf{y}):=\chi(|\mathbf{x}-\mathbf{y}|)\left[a_{f}(\mathbf{x}, \mathbf{y})-a_{g}(\mathbf{x}, \mathbf{y})\right] .
$$

We use the expansion (5.7) and (5.9), denoting $l$ by $l_{f}$, to indicate its dependence on $f$. We obtain

$$
\begin{aligned}
a_{1}(\mathbf{x}, \mathbf{y})= & \frac{i \kappa}{2 \pi} \frac{e^{i \kappa|\mathbf{x}-\mathbf{y}|}}{1+|\mathbf{x}-\mathbf{y}|^{2}}\{f(\mathbf{x})[f(\mathbf{y})-g(\mathbf{y})]+[f(\mathbf{x})-g(\mathbf{x})] g(\mathbf{y})\} J_{f}(\mathbf{y}) \\
& +\left(l_{f}(\mathbf{x}, \mathbf{y})-l_{g}(\mathbf{x}, \mathbf{y})\right) J_{f}(\mathbf{y}) \\
& +\left(\frac{i \kappa g(\mathbf{x}) g(\mathbf{y})}{2 \pi} \frac{e^{i \kappa|\mathbf{x}-\mathbf{y}|}}{1+|\mathbf{x}-\mathbf{y}|^{2}}+l_{g}(\mathbf{x}, \mathbf{y})\right)\left(J_{f}(\mathbf{y})-J_{g}(\mathbf{y})\right)
\end{aligned}
$$

for $\mathbf{x}, \mathbf{y} \in \mathbb{R}^{2}, \mathbf{x} \neq \mathbf{y}$.

The integral operator whose kernel is the first term of (5.21) can be bounded using Lemma 4.2 and (4.5). Similarly, the integral operator whose kernel is the last term of (5.21) can be bounded using Lemma 4.2, (4.5), (5.8), and (4.8), noting that Remark 5.3 guarantees the uniformity of (5.8) for $f \in B$. To bound the integral operator whose kernel is the second term of (5.21), we construct, for every $\eta \in(0,1)$, a function $\ell_{\eta} \in L^{1}\left(\mathbb{R}^{2}\right)$ such that

$$
\left|\left(l_{f}(\mathbf{x}, \mathbf{y})-l_{g}(\mathbf{x}, \mathbf{y})\right) J_{f}(\mathbf{y})\right| \leq \ell_{\eta}(\mathbf{x}-\mathbf{y}), \quad \mathbf{x}, \mathbf{y} \in \mathbb{R}^{2},
$$

whenever $f, g \in B$ and $\|f-g\|_{B C^{1, \alpha}\left(\mathbb{R}^{2}\right)}$ is sufficiently small, and such that $\left\|\ell_{\eta}\right\|_{L^{1}\left(\mathbb{R}^{2}\right)} \rightarrow$ 0 as $\eta \rightarrow 0$, and then we use the estimate (4.8). Together, the bounds on the three parts of $A_{1}$ show (5.19) for the global part of the operator.

The construction of $\ell_{\eta}$ is as follows. We choose (possible by Remark 5.3) a constant $C>0$ so that (5.8) holds for all $f \in B$. We choose another constant $C^{\prime}>0$ that is a bound for $\left\|J_{f}\right\|_{L^{\infty}\left(\mathbb{R}^{2}\right)}$ for $f \in B$. Then, where $\tilde{\ell} \in L^{1}\left(\mathbb{R}^{2}\right)$ is defined as in Lemma 5.2, we set

$$
\ell_{\eta}(\mathbf{y}):=\left\{\begin{array}{cc}
\eta^{3}, & \eta<|\mathbf{y}|<\eta^{-1} \\
2 C C^{\prime} \tilde{\ell}(\mathbf{y}) & \text { otherwise }
\end{array}\right.
$$


Clearly, this satisfies that $\left\|\ell_{\eta}\right\|_{L^{1}\left(\mathbb{R}^{2}\right)} \rightarrow 0$ as $\eta \rightarrow 0$. Since, for every $\eta \in(0,1)$, $\left|l_{f}(\mathbf{x}, \mathbf{y})-l_{g}(\mathbf{x}, \mathbf{y})\right| \rightarrow 0$ as $\|f-g\|_{B C^{1, \alpha}\left(\mathbb{R}^{2}\right)} \rightarrow 0$, uniformly in $f$ and $g$ for $f, g \in B$, and uniformly in $\mathbf{x}$ and $\mathbf{y}$ for $\eta \leq|\mathbf{x}-\mathbf{y}| \leq \eta^{-1}$, the bound (5.22) holds for all $f, g \in B$ with $\|f-g\|_{B C^{1, \alpha}\left(\mathbb{R}^{2}\right)}$ sufficiently small.

The local operator. For the local operator we argue in a similar way as for the global operator, in particular in a similar way as for the integral operator corresponding to the second term in (5.21). In particular, where $a_{2}$ is the kernel of the local operator, it holds for every $\eta>0$ that $\left|a_{2}(\mathbf{x}, \mathbf{y})\right| \rightarrow 0$ as $\|f-g\|_{B C^{1, \alpha}\left(\mathbb{R}^{2}\right)} \rightarrow 0$, uniformly in $f$ and $g$ for $f, g \in B$, and uniformly in $\mathbf{x}$ and $\mathbf{y}$ for $|\mathbf{x}-\mathbf{y}| \geq \eta$, and (5.5) takes the role of (5.8).

We have just established continuous dependence of the single- and double-layer potential operators on the boundary $\Gamma_{f}$. To show later that the limiting absorption condition (2.5) is satisfied in the case $\kappa>0$ we need to also establish continuous dependence on $\kappa$, which we do by similar arguments.

Lemma 5.7. Denote $S$ and $K$ temporarily by $S_{\kappa_{1}}$ and $K_{\kappa_{1}}$ to indicate their dependence on $\kappa_{1}$. Then, where $A_{\kappa_{1}}$ denotes either $S_{\kappa_{1}}$ or $K_{\kappa_{1}}$, it holds that

$$
\left\|A_{\kappa_{1}}-A_{0}\right\|_{L^{2}(\Gamma) \rightarrow L^{2}(\Gamma)} \rightarrow 0
$$

as $\kappa_{1} \rightarrow 0$.

Proof. As we did when proving Theorem 2.1 we split $A_{\kappa_{1}}$ into global and local parts, as $A_{\kappa_{1}}=A_{1}+A_{2}$, with $A_{1}, A_{2}$ defined by (5.2) and (5.3). As in the proofs of Lemma 5.1 and 5.2 we denote the kernel of $A_{j}$ by $a_{j}$.

To show (5.23) for the local part $A_{2}$ we note that $a_{2}(x, y)$ depends continuously on $\kappa_{1}$, uniformly in $x$ and $y$ for $|x-y| \geq \eta$ and every $\eta>0$, and that, by Remark 5.3 , the bound (5.5) holds uniformly in $\kappa_{1}$ for $\kappa_{1} \in[0,1]$. We then argue as for the local part in the proof of Theorem 5.6, showing that the kernel of the local part of $A_{\kappa_{1}}-A_{0}$ is bounded by an $L^{1}$ convolution kernel $\ell(\mathbf{x}-\mathbf{y})$ with $\|\ell\|_{L^{1}\left(\mathbb{R}^{2}\right)} \rightarrow 0$ as $\kappa_{1} \rightarrow 0$. Finally, we apply (4.8).

To show (5.23) for the global part $A_{2}$ we use the representation (5.7) for $a_{1}(x, y)$, which splits $a_{1}$ into a weakly singular part $l(\mathbf{x}, \mathbf{y})$, bounded by (5.8), and a strongly singular part $l^{*}(\mathbf{x}, \mathbf{y})$, given explicitly by (5.9) or (5.10). To show (5.23) for the weakly singular part of $A_{2}$ we argue exactly as we did in the proof of Theorem 5.6, noting that, by Remark 5.3, (5.8) holds uniformly in $\kappa_{1}$ for $\kappa_{1} \in[0,1]$, and that $l(\mathbf{x}, \mathbf{y})$ depends continuously on $\kappa_{1}$, uniformly in $\mathbf{x}$ and $\mathbf{y}$ for $\eta \leq|\mathbf{x}-\mathbf{y}| \leq \eta^{-1}$, for every $\eta \in(0,1)$. That (5.23) holds for the strongly singular part of $A_{2}$ follows from Lemma 4.2 and (4.5).

6. Uniqueness and existence results. In this section we prove, for the case when the surface is mildly rough, uniqueness and existence for our integral equation formulation and for the boundary value problem and the scattering problem defined in section 2. As the first step in this argument we prove Theorem 2.2 on the equivalence of the integral equation (2.16) and the boundary value problem.

Proof of Theorem 2.2. Let $v$ be the combined single- and double-layer potential $v$, defined in (2.13), with density $\varphi \in X$. By Theorem 5.5, $v \in C^{2}(D) \cap C(\bar{D})$ and satisfies the Helmholtz equation in $D$. Further, due to the jump relations (5.12) and (5.13), $v=g \in X$ on $\Gamma$ if and only if the density $\varphi$ satisfies the boundary integral equation (2.16). Applying Theorem 5.5 again, we see that $v$ satisfies the bound (2.4). This yields the equivalence statement for $\kappa_{1}>0$.

For real $\kappa$, in addition, we need to show the limiting absorption principle (2.5). 
Let $a(x, y)=\partial G(x, y) / \partial \nu(y)-i \eta G(x, y)$, so that

$$
v(x)=\int_{\Gamma} a(x, y) \varphi(y) d s(y), \quad x \in D .
$$

Suppose, as stated in the theorem, that $\varphi^{(\kappa+i \epsilon)} \in X$ satisfies the integral equation (2.16) with $\kappa$ replaced by $\kappa+i \epsilon$, for all sufficiently small $\epsilon>0$, and that $\| \varphi-$ $\varphi^{(\kappa+i \epsilon)} \|_{L_{2}(\Gamma)} \rightarrow 0$ as $\epsilon \rightarrow 0$. Let $a^{(\kappa+i \epsilon)}$ denote $a$ with $\kappa$ replaced with $\kappa+i \epsilon$, and define $v^{(\kappa+i \epsilon)}$ by $(6.1)$ with $a, \varphi$ replaced by $a^{(\kappa+i \epsilon)}, \varphi^{(\kappa+i \epsilon)}$, respectively. We have shown in the previous paragraph that $v^{(\kappa+i \epsilon)}$ satisfies Problem 2 (with $\kappa$ replaced by $\kappa+i \epsilon)$. To show the limiting absorption principle $(2.5)$ we need to show that $v^{(\kappa+i \epsilon)}(x) \rightarrow v(x)$ as $\epsilon \rightarrow 0$. We have

$$
\begin{aligned}
v^{(\kappa+i \epsilon)}(x)-v(x)= & \int_{\Gamma}\left(a^{(\kappa+i \epsilon)}(x, y)-a(x, y)\right) \varphi^{(\kappa+i \epsilon)}(y) d s(y) \\
& +\int_{\Gamma} a(x, y)\left(\varphi^{(\kappa+i \epsilon)}(y)-\varphi(y)\right) d s(y) .
\end{aligned}
$$

We see that the second term tends to zero as $\epsilon \rightarrow 0$ by the bound (5.15). Clearly, $a^{(\kappa+i \epsilon)}(x, y)-a(x, y) \rightarrow 0$ as $\epsilon \rightarrow 0$ for every $y \in \Gamma$. Thus, applying the CauchySchwarz inequality and then the dominated convergence theorem, noting that the bound (5.17) holds uniformly in $\kappa$, we see that the first term tends to 0 as $\epsilon \rightarrow 0$.

We obtain uniqueness of solution for the boundary value problem (proving Theorem 2.3) as follows. Due to [6, Theorem 1] (see also [24, Theorem 3.1]), a solution $u \in C^{2}(G) \cap C(\bar{G})$ to the Helmholtz equation (2.1) with $\operatorname{Im}(\kappa)>0$ on an open set $G \subset \mathbb{R}^{n}$ which satisfies the growth condition $|u(x)| \leq C e^{\theta|x|}$, with some constant $\theta<\operatorname{Im}(\kappa)$, and the boundary condition $u(x)=0$ for $x \in \partial G$ will vanish identically on $G$. This result directly implies uniqueness for the scattering problem and the boundary value problem for $\kappa_{1}>0$. For $\kappa_{1}=0$ uniqueness is a consequence of the limiting absorption principle we require, i.e., of the convergence (2.5).

Next we turn to establishing existence of solution in the mildly rough case. But first we prove a preliminary lemma which shows that to establish unique solvability of the integral equation in the space $X$ it is enough to study solvability in $L^{2}(\Gamma)$.

Lemma 6.1. Suppose that the integral equation (2.16) has exactly one solution $\varphi \in L^{2}(\Gamma)$ for every $g \in L^{2}(\Gamma)$. Then also (2.16) has exactly one solution $\varphi \in X$ for every $g \in X$, so that $(I+K-i \eta S)^{-1}$ exists and is bounded as an operator on $X$.

Proof. If the assumptions of the lemma hold, then (2.16) has exactly one solution $\varphi \in L^{2}(\Gamma)$ for every $g \in X \subset L^{2}(\Gamma)$. Further, defining $A=K-i \eta S$, it holds that $\varphi=A \varphi+2 g$ and, by induction, that, for every $n \in \mathbb{N}$,

$$
\varphi=A^{n} \varphi+2\left(A^{n-1}+\cdots+A^{0}\right) g .
$$

Now, by Theorem 2.1, $A$ is a bounded operator on $X$ and, by Corollary 5.4, $A^{n}$ is a bounded operator from $L^{2}(\Gamma)$ to $X$ for some $n \in \mathbb{N}$. Thus $\varphi \in X$. We have shown that (2.16) has exactly one solution $\varphi \in X$ for every $g \in X$, so that $(I+K-i \eta S)^{-1}$ exists as an operator on $X$. Since $X$ is a Banach space it follows as a standard corollary of the open mapping theorem that $(I+K-i \eta S)^{-1}$ is bounded.

As a corollary of Theorems 2.2 and 2.3 and Lemmas 5.7 and 6.1 we have the following result. 
Corollary 6.2. If $(I+K-i \eta S)^{-1}$ exists as a bounded operator on $L^{2}(\Gamma)$, then the boundary value problem and scattering problem have exactly one solution.

Proof. In the case $\kappa_{1}>0$ this result is clear from Theorems 2.2 and 2.3 and Lemma 6.1.

In the case $\kappa_{1}=0$ we note that, by Lemma 5.7 and standard operator perturbation arguments (e.g., [23]), if $(I+K-i \eta S)^{-1}$ exists as a bounded operator on $L^{2}(\Gamma)$ for $\kappa=\kappa_{0}>0$, then $(I+K-i \eta S)^{-1}$ exists and is a bounded operator on $L^{2}(\Gamma)$ for $\kappa=\kappa_{0}+i \kappa_{1}, 0 \leq \kappa_{1} \leq c$, for some $c>0$. Moreover, $(I+K-i \eta S)^{-1}$ depends continuously in the norm topology on $\kappa_{1}$ for $\kappa_{1} \in[0, c]$. Thus, provided $g \in L^{2}(\Gamma)$ depends continuously in norm on $\kappa_{1}$, for $\kappa_{1} \in[0, c]$, it holds that $(I+K-i \eta S)^{-1} g$ depends continuously in norm on $\kappa_{1} \in[0, c]$ in $L^{2}(\Gamma)$. If $g$ is given by (2.12), then, from the continuity of $\Phi(x, y)$ as a function of $\kappa_{1}$, uniformly in $x, y \in \mathbb{R}^{3}, x \neq y$, the bound (3.8), and the dominated convergence theorem, it follows that $g \in L^{2}(\Gamma)$ depends continuously in norm on $\kappa_{1}$ for $\kappa_{1} \in[0, c]$. Thus the result follows by Theorems 2.2 and 2.3 and Lemma 6.1 .

To make use of this result it remains to establish that $(I+K-i \eta S)$ is invertible as an operator on $L^{2}(\Gamma)$. We will show this first for the case of a flat surface $\Gamma_{h}=$ $\left\{y=(\mathbf{y}, h): \mathbf{y} \in \mathbb{R}^{2}\right\}$, with $h>0$. In this case the kernels of $K$ and $S$ depend only on the difference $\mathbf{x}-\mathbf{y}$, and thus, identifying $\Gamma_{h}$ with $\mathbb{R}^{2}$, the operators are convolution operators on $L^{2}\left(\mathbb{R}^{2}\right)$.

In terms of the function $W_{h}$ defined by (4.16), it follows from (3.9) that we can write the kernel of the double-layer potential operator as $P_{h}(\mathbf{x}-\mathbf{y})$, where $P_{h}(\mathbf{y}):=$ $p_{h}(|\mathbf{y}|)$ and

$$
p_{h}(r):=-\frac{i \kappa h}{\pi} \frac{e^{i \kappa \sqrt{r^{2}+4 h^{2}}}}{\left(\sqrt{r^{2}+4 h^{2}}\right)^{2}}+\frac{h}{\pi} \frac{e^{i \kappa \sqrt{r^{2}+4 h^{2}}}}{\left(\sqrt{r^{2}+4 h^{2}}\right)^{3}}=-\frac{\partial}{\partial h}\left\{W_{h}(r)\right\}, \quad r>0 .
$$

The kernel of the single-layer potential operator is $Q_{h}(\mathbf{x}-\mathbf{y})$, where $Q_{h}(\mathbf{y}):=q_{h}(|\mathbf{y}|)$ and

$$
q_{h}(r):=2\left\{\frac{1}{4 \pi} \frac{e^{i \kappa r}}{r}-\frac{1}{4 \pi} \frac{e^{i \kappa \sqrt{r^{2}+4 h^{2}}}}{\sqrt{r^{2}+4 h^{2}}}\right\}=2 W_{0}(r)-2 W_{h}(r), \quad r>0 .
$$

Hence, the integral equation (2.16) is transformed into

$$
\varphi(\mathbf{x})+\int_{\mathbb{R}^{2}}\left\{P_{h}(\mathbf{x}-\mathbf{y})-i \eta Q_{h}(\mathbf{x}-\mathbf{y})\right\} \varphi(\mathbf{y}) d \mathbf{y}=2 g(\mathbf{x}), \quad \mathbf{x} \in \mathbb{R}^{2} .
$$

To prove invertibility in $L^{2}\left(\Gamma_{h}\right)$ we compute the 2D Fourier transforms of $P_{h}$ and $Q_{h}$. From (4.13), for almost all $\mathbf{k} \in \mathbb{R}^{2}$, where $k:=|\mathbf{k}|$,

$$
\left(\mathcal{F} P_{h}\right)(\mathbf{k})=-\int_{0}^{\infty} \frac{\partial W_{h}(r)}{\partial h} J_{0}(k r) r d r .
$$

To evaluate this integral we reverse the order of integration and differentiation and use (4.17) to get that, for almost all $\mathbf{k} \in \mathbb{R}^{2}$,

$$
\left(\mathcal{F} P_{h}\right)(\mathbf{k})=-\frac{\partial}{\partial h}\left\{\frac{1}{4 \pi} \frac{e^{-2 h \sqrt{k^{2}-\kappa^{2}}}}{\sqrt{k^{2}-\kappa^{2}}}\right\}=\frac{1}{2 \pi} e^{-2 h \sqrt{k^{2}-\kappa^{2}}} .
$$

The interchange of integration and differentiation with respect to $h$ is certainly justified whenever $k>0$ and $k \neq \kappa$. For then the integral (4.17) is well defined, and, 
using (4.20), we see that for every $H>0$ there exists a constant $C>0$ such that

$$
\left|\frac{\partial W_{h}(r)}{\partial h} J_{0}(k r) r\right|=\left|\frac{\partial}{\partial h}\left\{\frac{1}{4 \pi} \frac{e^{i \kappa \sqrt{r^{2}+4 h^{2}}}}{\sqrt{r^{2}+4 h^{2}}}\right\} J_{0}(k r) r\right| \leq \frac{C}{r^{3 / 2}}
$$

for $r \geq 1$ and $0 \leq h \leq H$.

The Fourier transform of $Q_{h}$ can also be evaluated using (4.17). We obtain that

$$
\left(\mathcal{F} Q_{h}\right)(\mathbf{k})=\frac{1}{2 \pi}\left\{\frac{1}{\sqrt{k^{2}-\kappa^{2}}}-\frac{e^{-2 h \sqrt{k^{2}-\kappa^{2}}}}{\sqrt{k^{2}-\kappa^{2}}}\right\}
$$

for all $\mathbf{k} \in \mathbb{R}^{2}$ with $k \neq \kappa$. We combine the Fourier transforms of $P_{h}$ and $Q_{h}$ to derive for the Fourier transform of the kernel $R_{h}:=P_{h}-i \eta Q_{h}$ of $K-i \eta S$ the formula $\left(\mathcal{F} R_{h}\right)(\mathbf{k})=\hat{r}_{h}(k)$, for almost all $\mathbf{k} \in \mathbb{R}^{2}$, where

$$
\hat{r}_{h}(k):=\frac{1}{2 \pi}\left\{e^{-2 h \sqrt{k^{2}-\kappa^{2}}}-i \eta \frac{1-e^{-2 h \sqrt{k^{2}-\kappa^{2}}}}{\sqrt{k^{2}-\kappa^{2}}}\right\}, \quad k \geq 0 .
$$

From (4.2) we see that, for $\psi \in L^{2}\left(\mathbb{R}^{2}\right)$,

$$
(I+K-i \eta S) \psi=\mathcal{F}^{-1}\left(\left(1+2 \pi \mathcal{F} R_{h}\right)(\mathcal{F} \psi)\right) .
$$

Since $\mathcal{F}$ is an isomorphism on $L^{2}\left(\mathbb{R}^{2}\right)$ it follows that the inverse of $I+K-i \eta S$ exists as a bounded operator from $L^{2}\left(\Gamma_{h}\right)$ into $L^{2}\left(\Gamma_{h}\right)$ if and only if

$$
\text { ess. } \inf _{\mathbf{k} \in \mathbb{R}^{2}}\left|1+2 \pi\left(\mathcal{F} R_{h}\right)(\mathbf{k})\right|=\inf _{k \geq 0}\left|1+2 \pi \hat{r}_{h}(k)\right|>0 .
$$

We need to investigate $K(k):=1+2 \pi \hat{r}_{h}(k)=A\left(h \sqrt{k^{2}-\kappa^{2}}\right)$, for $k \geq 0$, where

$$
A(z):=1+e^{-2 z}-\frac{i h \eta}{z}\left(1-e^{-2 z}\right) .
$$

We recall that (see (4.17)) the square root is to be taken with $\sqrt{k^{2}-\kappa^{2}} \in V:=\{z \in$ $\mathbb{C}: \operatorname{Re} z \geq 0, \operatorname{Im} z \leq 0\}$. Indeed, in the case that $\kappa_{1}>0$, so that $\operatorname{Im}\left(k^{2}-\kappa^{2}\right)<0$, it is clear that $\sqrt{k^{2}-\kappa^{2}}$ lies in the interior of $V$. Now $A$ is an entire function (the singularity at 0 is removable) so that $K$ is continuous on $[0, \infty)$. Further, $K(k) \rightarrow 1$ as $k \rightarrow \infty$. Thus, to show (6.7) it is enough to show that $K(k) \neq 0$ for $k \geq 0$, which holds if $A(z) \neq 0$ for $z \in V$; indeed, in the case $\kappa_{1}>0$, we need only show that $A(z) \neq 0$ for all $z$ in the interior of $V$.

So suppose $\eta \geq 0$, and consider first the case when $z=z_{0}-i z_{1}$, with $z_{0}>0$, $z_{1} \geq 0$. It holds that

$$
A(z)=-i\left(1+e^{-2 z}\right)\left(\frac{h \eta \tanh z}{z}+i\right)
$$

and straightforward calculations yield

$$
\operatorname{Im}\left(\frac{\tanh z}{z}\right)=\frac{z_{0} \sin \left(2 z_{1}\right)+z_{1} \sinh \left(2 z_{0}\right)}{2\left[\sinh ^{2} z_{0}+\cos ^{2} z_{1}\right]\left(z_{0}^{2}+z_{1}^{2}\right)} \geq 0,
$$

since $|\sin t| \leq t \leq \sinh t$ for $t \geq 0$. Thus (6.7) holds if $\eta \geq 0$ and $\kappa_{1}>0$. 
In the case $\kappa_{1}=0$ we need to show, additionally, that $A(z) \neq 0$ when $z=-i z_{1}$ with $z_{1} \geq 0$, in order to establish that $A(z) \neq 0$ for all $z \in V$. Now $A(0)=2-2 i \eta h$ and, for $z_{1}>0$, from (6.8), $A\left(-i z_{1}\right)=2 \cos z_{1}-\frac{2 i h \eta}{z_{1}} \sin z_{1}$. Thus, provided $\eta>0$, $A\left(-i z_{1}\right) \neq 0$ for $z_{1} \geq 0$, so $A(z) \neq 0$ for $z \in V$. Thus (6.7) holds if $\eta>0$.

We have proven, in the case $\eta>0$ and in the case $\eta=0, \kappa_{1}>0$, that (6.7) holds, and thus we have shown the invertibility of $I+K-i \eta S$ and the boundedness of the inverse operator in $L^{2}\left(\Gamma_{h}\right)$. Thus we have established the solvability of (2.16) in the space $L^{2}(\Gamma)$ for flat surfaces. If $\Gamma_{f}$ is mildly rough, we may use a perturbation argument to show that the integral equation remains solvable. We state our result precisely in the following theorem.

Theorem 6.3. Suppose that $h>0$ and that either $\eta>0$ or $\eta=0$ and $\kappa_{1}>0$. Then, provided $\|f-h\|_{B C^{1, \alpha}\left(\mathbb{R}^{2}\right)}$ is sufficiently small (so that $\Gamma_{f}$ is sufficiently close to the flat surface $f \equiv h)$, it holds that $(I+K-i \eta S)^{-1}$ exists and is bounded as an operator on $L^{2}\left(\Gamma_{f}\right)$.

Proof. Let $A=I+K-i \eta S$, and then denote $A$ by $A_{f}$ to denote its dependence on $f$. With the help of the isomorphism $I_{f}: L^{2}\left(\Gamma_{f}\right) \rightarrow L^{2}\left(\mathbb{R}^{2}\right)$ defined by (5.18) we associate $A_{f}$ with the element $\tilde{A}_{f}=I_{f} A_{f} I_{f}^{-1}$ of the set of bounded linear operators on $L^{2}\left(\mathbb{R}^{2}\right)$. Now $\tilde{A}_{h}$ is invertible with bounded inverse, by our analysis above for the flat plane case. Moreover, by the continuity of $\tilde{A}_{f}$ with respect to $f$ as proven in Theorem 5.6 it follows from standard arguments that $\tilde{A}_{f}$ is boundedly invertible on $L^{2}\left(\mathbb{R}^{2}\right)$ for $\|f-h\|_{B C^{1, \alpha}\left(\mathbb{R}^{2}\right)}$ sufficiently small, and so $A_{f}$ is boundedly invertible on $L^{2}\left(\Gamma_{f}\right)$.

Combining Theorem 6.3 with Lemma 6.1 we deduce Theorem 2.4. Combining Theorem 6.3 with Corollary 6.2 we establish Theorem 2.5.

\section{REFERENCES}

[1] M. Abramowitz And I. A. Stegun, Handbook of Mathematical Functions, Dover, New York, 1965.

[2] T. ARENs, Existence of solution in elastic wave scattering by unbounded rough surfaces, Math. Methods Appl. Sci., 25 (2002), pp. 507-528.

[3] T. Arens, S. N. Chandler-Wilde, And K. O. Haseloh, Solvability and spectral properties of integral equations on the real line. II. $L^{p}$-spaces and applications, J. Integral Equations Appl., 15 (2003), pp. 1-35.

[4] H. Brakhage And P. Werner, Über das Dirichletsche Außenraumproblem für die Helmholtzsche Schwingungsgleichung, Arch. Math., 16 (1965), pp. 325-329.

[5] S. N. Chandler-Wilde and A. T. Peplow, A boundary integral equation formulation for the Helmholtz equation in a locally perturbed half-plane, ZAMM Z. Angew. Math. Mech., 85 (2005), pp. 79-88.

[6] S. N. ChandleR-Wilde AND C. R. Ross, Uniqueness results for direct and inverse scattering by infinite surfaces in a lossy medium, Inverse Problems, 11 (1995), pp. 1063-1067.

[7] S. N. Chandler-Wilde and C. R. Ross, Scattering by rough surfaces: The Dirichlet problem for the Helmholtz equation in a nonlocally perturbed half-plane, Math. Methods Appl. Sci., 19 (1996), pp. 959-976.

[8] S. N. Chandler-Wilde, C. R. Ross, and B. Zhang, Scattering by infinite one-dimensional rough surfaces, R. Soc. Lond. Proc. Ser. A Math. Phys. Eng. Sci., 455 (1999), pp. 37673787.

[9] S. N. Chandler-Wilde AND B. Zhang, Electromagnetic scattering by an inhomogenous conducting or dielectric layer on a perfectly conducting plate, R. Soc. Lond. Proc. Ser. A Math. Phys. Eng. Sci., 454 (1998), pp. 519-542.

[10] S. N. Chandler-Wilde And B. Zhang, A generalised collectively compact operator theory with an application to second kind integral equations on unbounded domains, J. Integral Equations Appl., 14 (2002), pp. 11-52.

[11] S. N. Chandler-Wilde, B. Zhang, and C. R. Ross, On the solvability of second kind integral equations on the real line, J. Math. Anal. Appl., 245 (2000), pp. 28-51. 
[12] W. C. Chew, J. M. Song, T. J. Cui, S. Velarnparambil, M. L. Hastriter, and B. Hu, Review of large scale computing in electromagnetics with fast integral equation solvers, CMES Comput. Model. Eng. Sci., 5 (2004), pp. 361-372.

[13] D. Colton And R. Kress, Inverse Acoustic and Electromagnetic Scattering Theory, 2nd ed., Springer-Verlag, Berlin, 1998.

[14] D. L. Colton And R. Kress, Integral Equation Methods in Scattering Theory, John Wiley, New York, 1983.

[15] J. A. DeSanto, Scattering by rough surfaces, in Scattering: Scattering and Inverse Scattering in Pure and Applied Science, R. Pike and P. Sabatier, eds., Academic Press, San Diego, 2002, pp. 15-36.

[16] J. A. DeSanto And P. A. Martin, On the derivation of boundary integral equations for scattering by an infinite two-dimensional rough surface, J. Math. Phys., 39 (1998), pp. 894912.

[17] D. Dobson and A. Friedman, The time harmonic Maxwell equations in a doubly-periodic structure, J. Math. Anal. Appl., 166 (1992), pp. 507-528.

[18] A. Erdélyi, W. Magnus, F. Oberhettinger, and F. G. Tricomi, Tables of Integral Transforms, vol. II, McGraw-Hill, London, 1954.

[19] R. KRess And T. TRAn, Inverse scattering for a locally perturbed half-plane, Inverse Problems, 16 (2000), pp. 1541-1559.

[20] W. McLean, Strongly Elliptic Systems and Boundary Integral Equations, Cambridge University Press, Cambridge, UK, 2000.

[21] J.-C. Nedelec And F. Starling, Integral equation methods in a quasi-periodic diffraction problem for the time-harmonic Maxwell's equations, SIAM J. Math. Anal., 22 (1991), pp. 1679-1701.

[22] J. A. OGilvy, Theory of Wave Scattering from Random Rough Surfaces, Adam Hilger, Bristol, UK, 1991.

[23] M. Reed and B. Simon, Methods of modern mathematical physics: Part II. Fourier Analysis, Self-Adjointness, Academic Press, New York, 1975.

[24] C. R. Ross, Direct and Inverse Scattering by Rough Surfaces, Ph.D. thesis, Brunel University, Uxbridge, UK, 1996.

[25] M. Saillard and A. Sentenac, Rigorous solutions for electromagnetic scattering from rough surfaces, Waves Random Media, 11 (2001), pp. R103-R137.

[26] R. H. TorRes And G. V. Welland, The Helmholtz-equation and transmission problems with Lipschitz interfaces, Indiana Univ. Math. J., 42 (1993), pp. 1457-1485.

[27] L. Tsang, C. H. Chan, K. PaK, And H. SAngani, Monte-Carlo simulations of large-scale problems of random rough surface scattering and applications to grazing incidence with the BMIA/canonical grid method, IEEE Trans. Antennas and Propagation, 43 (1995), pp. 851-859.

[28] A. G. Voronovich, Wave Scattering from Rough Surfaces, 2nd ed., Springer, Berlin, 1998.

[29] K. F. WARNICK AND W. C. CHEW, Numerical simulation methods for rough surface scattering, Waves Random Media, 11 (2001), pp. R1-R30.

[30] A. Willers, The Helmholtz equation in disturbed half-spaces, Math. Methods Appl. Sci., 9 (1987), pp. 312-323.

[31] M. Xia, C. H. Chan, S. Li, B. Zhang, and L. Tsang, An efficient algorithm for electromagnetic scattering from rough surfaces using a single integral equation and multilevel sparse-matrix canonical-grid method, IEEE Trans. Antennas and Propagation, 51 (2003), pp. 1142-1149.

[32] B. Zhang and S. N. Chandler-Wilde, Integral equation methods for scattering by infinite rough surfaces, Math. Methods Appl. Sci., 26 (2003), pp. 463-488. 